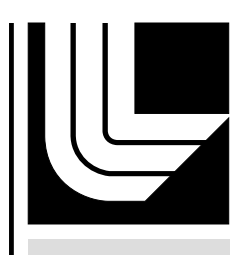

LAWRENCE LIVERMORE N A TION A L LABORATORY

\title{
Ab initio Guided Optimization of GaTe for Radiation Detection Applications
}

C. Rocha Leao, V. Lordi

April 14, 2011

Physical Review B 
This document was prepared as an account of work sponsored by an agency of the United States government. Neither the United States government nor Lawrence Livermore National Security, LLC, nor any of their employees makes any warranty, expressed or implied, or assumes any legal liability or responsibility for the accuracy, completeness, or usefulness of any information, apparatus, product, or process disclosed, or represents that its use would not infringe privately owned rights. Reference herein to any specific commercial product, process, or service by trade name, trademark, manufacturer, or otherwise does not necessarily constitute or imply its endorsement, recommendation, or favoring by the United States government or Lawrence Livermore National Security, LLC. The views and opinions of authors expressed herein do not necessarily state or reflect those of the United States government or Lawrence Livermore National Security, LLC, and shall not be used for advertising or product endorsement purposes. 


\title{
$A b$ initio guided optimization of GaTe for radiation detection applications
}

\author{
Cedric R. Leão* and Vincenzo Lordi ${ }^{\dagger}$ \\ Lawrence Livermore National Laboratory, Livermore, CA, 94550, USA
}

\begin{abstract}
The development of semiconductor-based radiation detectors that display high energy resolution while operating at room temperature is a pressing need for both scientific applications as well as homeland security. Practice has proven that the real performance of materials in such applications is often hindered by intrinsic defects and accidental impurities. Experimental efforts to improve the properties of such materials are both time consuming and costly, since they rely largely on trial and error. In this paper, the properties of gallium telluride (GaTe) — a high Z, moderate band gap semiconductor-are investigated for room-temperature radiation detection applications. Systematic theoretical modeling based on density functional theory calculations is used to suggest experimental processes to grow this semiconductor with optimal properties, by judiciously identifying the most detrimental native defects and devising ways to minimize their occurrence as well as compensating their electronic impact on the crystal. The analysis suggests that material grown Ga-rich would have significantly higher resistivity, carrier mobilities, and carrier lifetimes compared to Te-rich material. In addition, Ge doping and In doping can be effective for carrier compensation of the material. Doping with Ge can be especially effective, if the ambipolar nature of substitutional incorporation on both $\mathrm{Ga}$ and Te sites is exploited.
\end{abstract}

PACS numbers: 61.72.Bb, 71.55.Ht, 72.20.Jv, 61.50.Ah

\section{INTRODUCTION}

Among the plethora of applications of semiconductors in electronic devices, the fabrication of radiation detectors is one in which the presence of free carriers is not desirable. The operation of such detectors relies on the ability to precisely measure photo-induced current in the material, enabling the inference of the energy of the incident radiation. For this purpose, background free carriers in the material add noise to the desired signal. Therefore, materials with high resistivity, $\gtrsim 10^{10} \Omega$-cm, are sought. In semiconductors, the main source of these undesired free electrons or holes are crystalline point defects and impurities. Not only may such defects add free carriers to the material, but they also disturb its periodic potential, which can result in significant scattering of carriers flowing in an electric current, reducing their mobilities. Moreover, native defects and impurities can introduce semi-filled levels in the band gap. These levels can accept or donate electrons to the host material, acting as carrier trapping centers, reducing the effective lifetimes of the photo-excited carriers. The detector energy resolution, which is sought to be maximized, is degraded if incomplete carrier collection occurs in the device from the carrier drift length (mobility $\times$ lifetime $\times$ electric field, or $\mu \tau E$ ) being too short compared to the physical dimension of the device. For detection of gamma rays and X-rays, absorption depths are on the order of a centimeter for most semiconductors, so an ideal value of the $\mu \tau$ figure of merit is on the order of $10^{-2} \mathrm{~cm}^{2} / \mathrm{V}$ or greater. ${ }^{1,2}$

Currently, two semiconductors are dominant for applications in radiation detectors. The first is germanium, a material which routinely can be grown with high purity and extreme crystallinity, in addition to presenting extremely high carrier mobilities. The disadvantages of $\mathrm{Ge}$ are that it possesses a relatively low atomic number $(Z)$, which leads to a long absorption depth, and a relatively small band gap, which requires cryogenic cooling to achieve low-noise operation. The requirement for cryogenic cooling limits large scale applications and increases the cost of Ge-based devices. ${ }^{3}$ The other preva- lent material is $\mathrm{Cd}_{x} \mathrm{Zn}_{1-x} \mathrm{Te}(\mathrm{CZT})$, which has a band gap large enough for low-noise operation at room-temperature. However, the addition of ( $10 \mathrm{at} \%$ ) $\mathrm{Zn}$ to CdTe to form the CZT alloy introduces significant internal strains to the lattice, making the growth of large defect-free single crystals of CZT difficult. Often, only small grains can be harvested from a grown boule, manually selected to exclude portions of the crystal containing extended defects. For a high performance CZT detector, many such small pieces must be harvested and combined to obtain sufficient volume for efficient absorption of radiation, leading to a very high material cost (often prohibitive for high-volume applications). ${ }^{1,2}$

Thus, the pursuit of new materials for efficient, highresolution radiation detectors operating at room-temperature has been intense. The criteria for a high-performance semiconductor material are: (a) high $Z$, (b) a band gap sufficiently large relative to thermal excitation of carriers $(\gtrsim 1.6 \mathrm{eV}),(\mathrm{c})$ high carrier mobilities, (d) long carrier lifetimes, and (e) the possibility to grow the material with high crystallinity and purity. Within these criteria, gallium telluride $(\mathrm{GaTe})$ is a possible strong candidate, with a band gap of $\sim 1.7 \mathrm{eV} 4$ and also a relatively high average $Z$ of 45 , with $Z_{\mathrm{Ga}}=31$ and $Z_{\mathrm{Te}}=52$. Several groups, however, have reported that as-grown material appears always to be $p$-type, with high free carrier concentrations $\left(\sim 10^{16}-10^{17} \mathrm{~cm}^{-3}\right)$, low resistivities $(\sim 20-200 \Omega-\mathrm{cm})$, and very low carrier mobilities $\left(\sim 10-60 \mathrm{~cm}^{2} / \mathrm{V}-\mathrm{s}\right){ }^{4-9}$

In the report, we describe the use of a fully ab-initio theoretical tool set ${ }^{10-12}$ to analyze how intrinsic defects and accidental impurities hinder the performance of GaTe as a roomtemperature radiation detector, and how possible compensation dopants behave to improve the properties. In Sect. II, we discuss the computational methodology. In Sect. III A, we evaluate which intrinsic defects are prevalent under different growth conditions and determine their electronic nature as either acceptors or donors and any deep levels they introduce. The computational results are assessed against experimental data. In the Sect. III B, we analyze the effects of two likely impurities, $\mathrm{O}$ and $\mathrm{H}$, on the electronic structure of 
the material. In Sect. IIIC, we study two possible compensation dopants for GaTe: In, which may diminish the occurrence of Ga vacancies; and Ge, which may counteract the excess of holes consistently reported for as-grown material. The net excess carrier concentrations in the material for the various cases we consider are determined self consistently and reported in Sect. IIIE. The results indicate that the pristine material should be $n$-type over most of the allowed range of growth conditions. However, our analysis shows that the introduction of impurities, particularly $\mathrm{H}$, even in relatively low concentrations can turn the material $p$-type, in agreement with experiments. We further show that Ge doping can be effective for compensating the free carriers in GaTe, resulting in higher resistivity material, particularly when combined with a careful annealing prescription. Finally, in Sect. III F, we analyze the impact of each considered defect or dopant on charge transport (carrier mobility) in the material. We find that the dominant Ge- and In-related defects are much weaker carrier scatterers than some of the most frequent intrinsic defects in GaTe, making these elements desirable dopants. In Sect. IV, the whole of the results are considered in terms of the positive and negative effects of each considered defect on the detection performance of GaTe. The most detrimental defects and impurities are identified, and the efficacy of each dopant is considered in light of any possible negative effects on device performance. Finally, in Sect. V, we summarize our results and present suggestions for crystal growth processes to produce higher quality material for radiation detection applications.

\section{CALCULATION DETAILS}

Calculations were performed using density functional theory $(\mathrm{DFT})^{13,14}$ within the local density approximation $(\text { LDA })^{15}$ and the supercell approximation. Core electrons were treated with the projector-augmented wave (PAW) method, ${ }^{16,17}$ as implemented in the Vienna $A b$ Initio Simulation (VASP) code. ${ }^{18-21}$ Valence electrons were represented with planewaves using an energy cutoff of $380 \mathrm{eV}$; for calculations involving oxygen atoms, a planewave cutoff of $500 \mathrm{eV}$ was employed. Structural optimizations were performed using a conjugate gradients algorithm until the HellmannFeynman forces on all atoms were less than $10^{-2} \mathrm{eV} / \AA$.

GaTe presents a layered and highly anisotropic structure, with a monoclinic primitive cell composed of $6 \mathrm{Ga}-\mathrm{Te}$ units. ${ }^{22}$ Brillouin zone (BZ) sampling was performed using the tetrahedron method with 88 irreducible points in the primitive cell $(7 \times 7 \times 6$ sampling $)$. Our tests indicated that after full atomic and volumetric relaxations, the LDA reproduces the measured crystal structure, including the interlayer spacing, associated to the lattice vector $\mathbf{c}$ indicated in table I with errors of less than $3 \%$. Calculations using the PBE generalized gradient approximation $(\mathrm{GGA})^{23}$ resulted in errors greater than $7 \%$ for the interlayer spacing (see Table I).

The LDA calculated band structure for bulk GaTe, shown in Fig. 1, also reproduces the main features of the experimental data, including a direct band gap at the $Z$ point of
TABLE I: GaTe structural parameters for different calculations. The primitive vectors $\mathbf{a}$ and $\mathbf{b}$ are contained on the layer plane and $\mathbf{c}$ is across the layers. Bond lengths and distances are given in $\AA$, the angle $\gamma$ formed between the vector $\mathbf{c}$ and the plane formed by vectors $\mathbf{a}$ and $\mathbf{b}$ is given in degrees, and $\varepsilon$ represents the percent discrepancies between the theoretical and experimental data.

\begin{tabular}{cccccc}
\hline \hline & Experimental $^{a}$ & GGA & $\varepsilon_{\mathrm{GGA}}(\%)$ & LDA & $\varepsilon_{\mathrm{LDA}}(\%)$ \\
\hline $\mathrm{Ga} 1-\mathrm{Ga} 2$ & 2.43 & 2.46 & 1.23 & 2.39 & 1.65 \\
$\mathrm{Ga} 3-\mathrm{Ga} 3$ & 2.44 & 2.48 & 1.64 & 2.41 & 1.23 \\
$\mathrm{Ga} 1-\mathrm{Te} 1$ & 2.64 & 2.69 & 1.89 & 2.64 & $\approx 0$ \\
$\mathrm{Ga} 3-\mathrm{Te} 3$ & 2.69 & 2.72 & 1.12 & 2.67 & 0.74 \\
$\mathbf{a}$ & 23.76 & 23.97 & 0.88 & 23.43 & 1.39 \\
$\mathbf{b}$ & 4.068 & 4.14 & 1.77 & 4.06 & 0.2 \\
$\mathbf{c}$ & 10.46 & 11.26 & 7.65 & 10.25 & 2.01 \\
$\gamma$ & 45.4 & 48.65 & 7.16 & 44.50 & 1.98 \\
\hline \hline
\end{tabular}

${ }^{a}$ Ref. 22

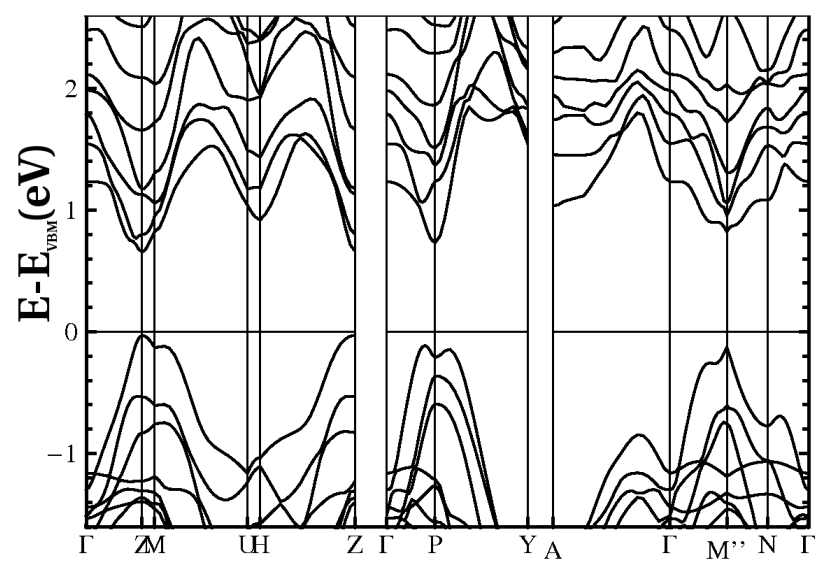

FIG. 1: DFT-LDA band structure of GaTe. The energy of the valence band maximum has been set to $0 \mathrm{eV}$.

the BZ, as previously reported. ${ }^{24}$ (Including spin-orbit effects does not significantly alter the band structure, as also previously reported, ${ }^{25}$ so we neglect it here.) The calculated band gap is $0.68 \mathrm{eV}$, significantly below the experimental value of $1.8 \mathrm{eV},{ }^{4,9,24}$ as expected for LDA.

The Brillouin zone for GaTe is shown in Fig. 2. The band edges coincide with the high symmetry point $Z$. The direction $Z-\Gamma$ corresponds to the direction across the atomic layers, perpendicular to the projection plane in Fig. 3. The directions $Z-M$ and $Z-H$, respectively, correspond to the two in-layer directions indicated by the $\mathrm{Y}$ and $\mathrm{X}$ axes in Fig. 3.

Previous experimental work on other layered materials, such as GaSe, has established that unexpected anisotropy of the effective masses of carriers often is found, with values as much as four times smaller across the layer planes than within them. ${ }^{26}$ For GaSe, this is the case for holes, whereas for electrons the effective mass in the layer plane is about three times smaller than perpendicular to it. Experimental results for the effective mass of holes in GaTe point to similar effects: $m_{h \perp}^{*}=1.0$ and $m_{h \|}^{*}=0.2$ were reported in Ref. 27, while $m_{h \perp}^{*}=0.995$ and $m_{h \|}^{*}=0.46$ were reported in Ref. 9 . Neither of these works report measured effective masses of 


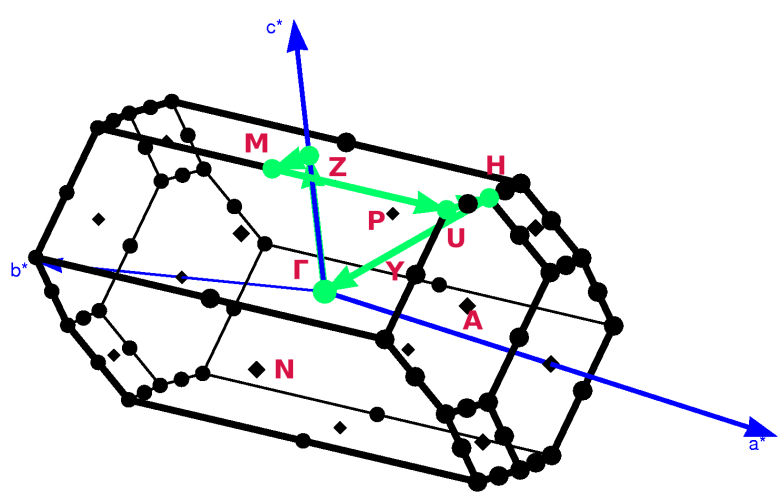

FIG. 2: (Color online) Brillouin zone of GaTe with the reciprocal lattice vectors and high symmetry points indicated.

TABLE II: Calculated effective masses, in units of $m_{0}$, for electrons $\left(m_{e}^{*}\right)$ and holes $\left(m_{h}^{*}\right)$ in GaTe in different crystallographic directions, using the LDA.

\begin{tabular}{ccc}
\hline \hline BZ direction (see Fig. 2) & $m_{e}^{*}$ & $m_{h}^{*}$ \\
\hline$Z-\Gamma$ (across atomic layers) & 0.160 & 0.123 \\
$Z-M$ (in-layer, along Y in Fig. 3) & 0.198 & 0.166 \\
$Z-H$ (in-layer, along X in Fig. 3) & 0.132 & 0.746 \\
\hline \hline
\end{tabular}

electrons. They also do not distinguish between the unequal directions within the layer planes of GaTe (e.g., axes X and $\mathrm{Y}$ in Fig. 3).

Given the two different orientations of the Ga-Ga bonds in the structure of GaTe, it is reasonable to expect significant differences in the transport of carriers even in different in-plane directions. Indeed, we find that is the case, particularly for holes, as shown by the effective masses listed in Table II, which were extracted from the calculated band structure. Our calculated results show that along the $Z-M$ direction (in the planes, along the horizontal Ga-Ga bonds), holes have an effective mass almost 6 times smaller than in the other in-plane direction $Z-H$ (perpendicular to the $\mathrm{Ga}-\mathrm{Ga}$ bonds). The hole effective mass across the layers is the lower than both in-plane directions, in general agreement with the experiments. Electrons show only a weak anisotropy of the effective masses. One might simplistically deduce the anisotropy of carrier mobilities in the different directions by comparing the effective masses, however for the across-plane direction the mobility apparently is limited by scattering off homopolar optical phonons, ${ }^{27}$ leading to relatively lower carrier mobilities in this direction. In any case, one must choose carefully and control were to place electrical contacts with respect to the crystallographic directions when fabricating devices for electrical measurements of $\mathrm{GaTe}$, and ultimately for fabricating detectors.

For the study of point defects in the GaTe structure, we used a supercell composed of 144 atoms, with dimensions $24.23 \times 23.40 \times 10.26 \AA$, and the $a$ and $b$ supercell lattice vectors aligned with the layer plane (see Fig. 3). Figure 3 also indicates the possible defect incorporation sites. Note that the stacking of the layers is tilted relative to the $z$-axis

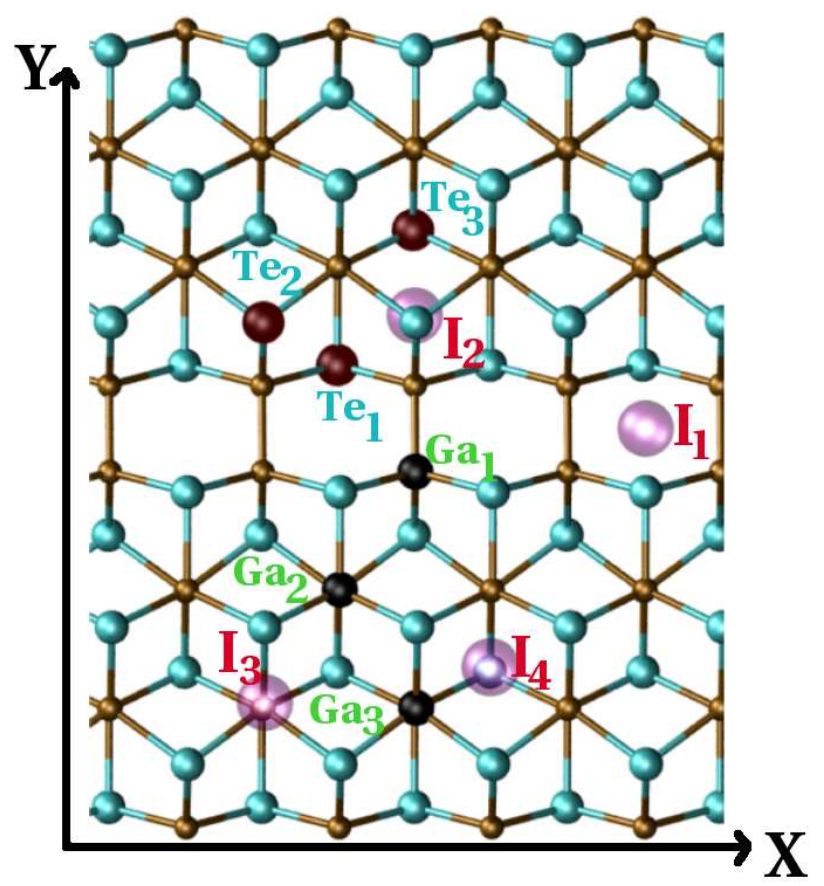

FIG. 3: (Color online) GaTe supercell with main defect incorporation sites indicated. Ga atoms are small brown spheres and Te atoms are large light blue spheres. Different substitutional sites are highlighted in red and green for $\mathrm{Ga}$ and Te, respectively, and labeled 1 and 3. Interstitial sites are indicated by labels $I_{1}-I_{4}$. Note that sites $I_{2}$ and $I_{4}$ differ in that $I_{2}$ is within the atomic layer while $I_{4}$ is over it.

indicated on the figure. For this supercell, we used 28 irreducible points $(3 \times 3 \times 6$ mesh $)$ for the Brillouin zone sampling with the tetrahedron method. Finite-size errors were checked against a 192-atom supercell containing two unit layers (dimensions $16.18 \times 23.42 \times 20.53 \AA$ ), for the case of a Te vacancy; differences in defect formation energy and intra-gap charge transition levels between the two supercells were of the order $10^{-2} \mathrm{eV}$. We preferred the use of 144-atom supercell for the bulk of the work presented in this paper due to its greater uniformity.

Charged defects were studied by adding or removing electrons from the supercell and adding a compensating uniform background charge to ensure charge neutrality of the periodic system (by the usual approach of nullifying the $G=0$ term of the potential energy Fourier transform). Spurious electrostatic interactions between the charged defect and its periodic images were analyzed to first order with the Makov-Payne monopole-monopole term: ${ }^{28}$

$$
E_{M P}=-\frac{q^{2} \alpha}{2 L \varepsilon},
$$

where $q$ is the charge state of the defect under consideration, $\alpha$ is the madelung constant of the supercell ( 0.9 for the cell of Fig. 3), $L$ is the distance between image defects (in our case, $24 \AA$ for the largest distance and $10 \AA$ for the shortest one) and $\varepsilon$ is the dielectric permittivity of the material $\left(7.7\right.$ for $\left.\mathrm{GaTe}^{9}\right)$. We note that the large size of the supercells used in this work lead to corrections in the total energy from Eq. (1) of less than 
$25 \mathrm{meV}$ for doubly-ionized defects, which is practically negligible.

\section{RESULTS AND DISCUSSION}

\section{A. Intrinsic defects}

\section{Structure and electronic character}

The low-symmetry and anisotropic structure of GaTe requires consideration of several nonequivalent defect incorporation sites, which are highlighted within a supercell in Fig. 3. In general, the unit cell contains six atoms, with three symmetry inequivalent $\mathrm{Ga}$ and $\mathrm{Te}$ sites each. The substitutional sites are highlighted in black and dark red for $\mathrm{Ga}$ and Te, respectively. The Ga sites indicated by 1 and 3 are qualitatively different by involving either in-plane Ga-Ga bonds (1) or perpendicular $\mathrm{Ga}-\mathrm{Ga}$ bonds (3). We refer to the in-plane $\mathrm{Ga}-$ $\mathrm{Ga}$ bonds are "horizontal" and perpendicular $\mathrm{Ga}-\mathrm{Ga}$ bonds as "vertical" in the remainder of this text. The two different Te sites are categorized by the number of bonds made to each type of Ga atom: site 1 has two bonds to "horizontal" $\mathrm{Ga}$ atoms and one bond to a "vertical" $\mathrm{Ga}$ atom, while site 3 has all three bonds to "vertical" Ga atoms. Two additional substitutional sites, labeled 2, correspond to Te atoms making one bond to horizontal $\mathrm{Ga}$ atoms, as well as vertical $\mathrm{Ga}$ atoms with no bonds to Te atoms on site 3.(See also Fig. 4.) Defects on sites 2 were not explicitly calculated for most cases, since their characteristics are similar to sites 3 . Figure 4 illustrates the qualitative electronic difference between site 1 and sites 2 and 3, for both Ga and Te sites, by showing the atomprojected local density of states at the band edges ( $Z$ point in the Brillouin zone $\left.{ }^{24}\right)$. A clear distinction of site 1 , with a small contribution to the formation of the band-edge states, and the similarity between sites 2 and 3 is apparent. Thus, we can expect different behavior of substitutional defects incorporated on the different sites, which we will show below to be the case, markedly for some defects. Finally, four different interstitial sites, which can be occupied by either Ga or Te atoms, are also indicated in Fig. 3 by translucent circles labeled $\mathrm{I}_{1}$ to $\mathrm{I}_{4}$.

We calculate the equilibrium concentration of each intrinsic point defect from its formation energy, $\Delta E_{f}$, which for moderate pressures and temperatures is given by ${ }^{29}$

$$
\Delta E_{f}=E_{d}-\left(E_{p}-n \mu_{x}+m \mu_{y}\right)+q\left(\mu_{e}+E_{\mathrm{VBM}}\right),
$$

where $E_{d}$ and $E_{p}$ are the total energies of the defective and the pristine supercells, respectively, $n(m)$ is the number of atoms of element $x(y)$ removed from (added to) the supercell and added to (removed from) a reservoir whose chemical potential is $\mu_{x}\left(\mu_{y}\right), q$ is the amount of charge exchanged from a reservoir with electron chemical potential $\mu_{e}$, which we reference to the energy of the valence band maximum, $E_{\mathrm{VBM}}$.

The energies of the atomic chemical reservoirs in equilibrium with the crystal depend heavily on the growth conditions. However, we can set limits on their variation from the following requirements ${ }^{11,29,30}$ : (i) the atomic chemical potential
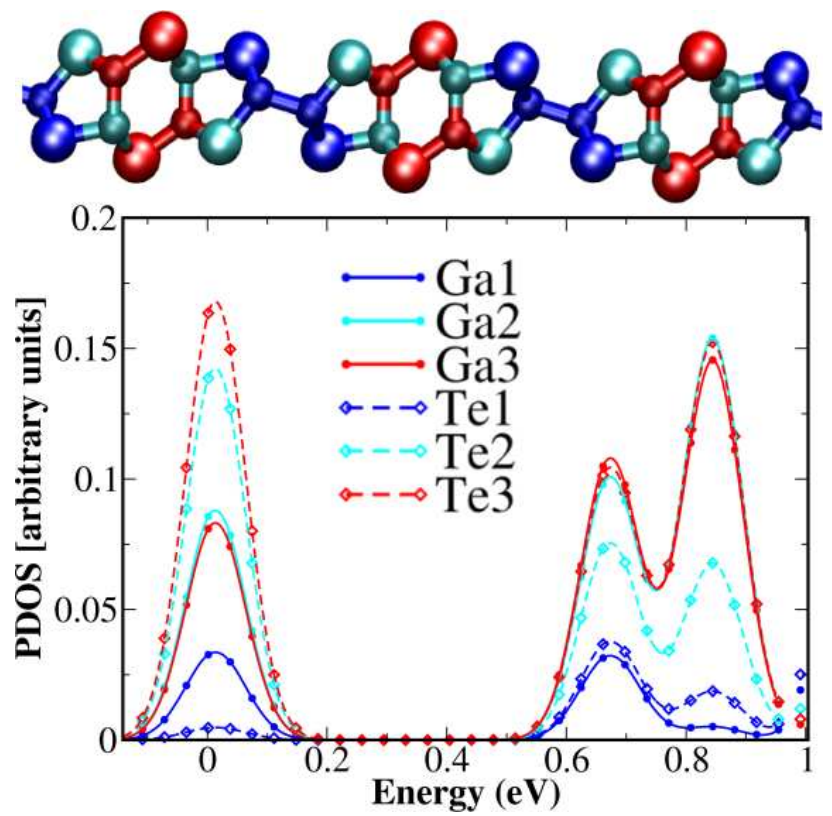

FIG. 4: (Color online) Density of states at the $Z$ point of the Brillouin zone, projected on each of the inequivalent atoms of the primitive cell of GaTe. On top, we show an edge-on view of one atomic layer of the crystal, with $\mathrm{Ga}$ atoms represented by smaller spheres and $\mathrm{Te}$ atoms by larger ones. Different colors correspond to the different sites (refer to the text), as labeled in the key. The top of the valence band was set at $0 \mathrm{eV}$ and a Gaussian smearing of $0.2 \mathrm{eV}$ was used.

in the compound is always lower (i.e., more negative) than the element's bulk potential, (ii) the heat of formation of the compound, $\Delta H_{f}$, equals the difference between the elemental chemical potentials in the compound and their upper limits (i.e., their bulk values), and (iii) there can be the occurrence of competing phases involving the constituent elements and also possibly impurities (e.g., $\mathrm{Ga}_{2} \mathrm{Te}_{3}, \mathrm{GaInTe}, \mathrm{GeTe}$, etc.). Symbolically, we have:

$$
\begin{gathered}
\mu_{x} \leq \mu_{x}^{B} \\
\mu_{x}+\mu_{y}=\Delta H_{f}+\mu_{x}^{B}+\mu_{y}^{B} \\
\mu_{x y} \leq \mu_{x_{2} y_{3}}, \mu_{x y} \leq \mu_{x y z}, \ldots
\end{gathered}
$$

where $\mu_{x}$ and $\mu_{x}^{B}$ represent the chemical potential of constituent $x$ in the compound and in the bulk, respectively. The compound heat of formation is given by

$$
\Delta H_{f}=\mu_{x y}-\mu_{x}^{B}-\mu_{y}^{B} .
$$

The equilibrium concentration of a defect is then given by

$$
c=c_{0} \exp \left(-\frac{E_{f}}{k_{B} T}\right),
$$

where $c_{0}$ is the concentration of possible sites for the defect, $k_{B}$ is Boltzmann's constant, and $T$ is absolute temperature.

The formation energy of each intrinsic defect is calculated using Eq. (2), for each possible incorporation site. The conditions of Eq. (3) are applied to compute the formation energies 
under conditions of stoichiometry (equal amounts of $\mathrm{Ga}$ and Te atoms to react, or $\mu_{\mathrm{Ga}}=\mu_{\mathrm{Te}}=\Delta H_{f} / 2$ ), the Ga-rich limit $\left(\mu_{\mathrm{Ga}}=\mu_{\mathrm{Ga}}^{B}\right.$ and $\left.\mu_{\mathrm{Te}}=0\right)$, or the Te-rich limit $\left(\mu_{\mathrm{Te}}=\mu_{\mathrm{Te}}^{B}\right.$ and $\mu_{\mathrm{Ga}}=0$ ). The results for the most important defects (lowest formation energies) are shown in Fig. 5. Data for intrinsic interstitials on sites $I_{2}, I_{3}$, and $I_{4}$ are excluded from Fig. 5, since they have significantly higher formation energy than interstitials on site $I_{1}$ (between 1 and $0.37 \mathrm{eV}$ for Te and 0.8 and $0.2 \mathrm{eV}$ for $\mathrm{Ga}$ ). We also haven't observed qualitatively different electronic behavior between interstitials on these sites and on site $I_{1}$.

The formation energies of the charged intrinsic defects in GaTe as a function of $\mu_{e}$ are displayed in Fig. 5. For clarity, only Fig. 5(b) shows all of the defects studied, while Fig. 5(a) and (b) only show the six lowest formation energy defects for the respective chemical condition. We note that the variations in the chemical environment ( $\mu_{\mathrm{Ga}}$ and $\mu_{\mathrm{Te}}$ ) only shift the formation energy curves up or down, but do not change the positions of the charge state transitions indicated by symbols in the Fig. 5.

We observe a general trend that antisites on positions labeled 1 and interstitials on positions labeled $I_{1}$ in Fig. 3 present significantly lower formation energy than the corresponding defects on sites labeled 3 or $I_{2}$. The reason is that sites 1 are closer to the domain of horizontally aligned $\mathrm{Ga}-\mathrm{Ga}$ bonds, where the lattice is more open and can more easily accommodate defects with increased volume. This phenomenon is shown in Fig. 6, where the relaxed structures of Te and Ga interstitials on sites $I_{1}$ and $I_{2}$ are compared. The increased lattice distortion and local strain in the $I_{2}$ interstitials is apparent, while the $I_{1}$ interstitials relax into the open space around the horizontal $\mathrm{Ga}-\mathrm{Ga}$ bonds. Similarly, interstitials on site $I_{4}$ have significantly lower formation energy than on site $I_{3}$ because in the former case the impurities relax into the open region of horizontal $\mathrm{Ga}-\mathrm{Ga}$ bonds. For vacancies, the trend is the opposite, since these are negative formation volume defects. Thus, the formation energies of $V_{\mathrm{Ga}}$ and $V_{\mathrm{Te}}$ are lower on site 3 than on site 1 , since the atomic packing is higher around site 3 and a vacancy generates less structural deformation than on the emptier region of site 1 .

Figure 5(a) shows that for Ga-rich conditions, the dominant defect for intrinsic material and across most all of the band gap is the positively charged $\mathrm{Ga}$ interstitial $\mathrm{Ga}_{i}^{1+}$. For $n$-type material with the Fermi level in the upper part of the band gap, negatively charged $\mathrm{Ga}$ antisites $\mathrm{Ga}_{\mathrm{Te}}$ are dominant. For Te-rich conditions shown in Fig. 5(c), a delicate balance exists among the predominant defects. The $\mathrm{Ga}$ interstitial $\mathrm{Ga}_{i}^{1+}$ dominates for highly $p$-type conditions, while $\mathrm{Te}_{i 1}^{0}, \mathrm{Te}_{\mathrm{Ga}}^{1-}$, and $V_{\mathrm{Ga} 3}^{1-}$ all have low formation energies near intrinsic conditions. For highly $n$-type material, the doubly negative $\mathrm{Ga}$ vacancy $V_{\mathrm{Ga} 3}^{2-}$ is dominant. Under stoichiometric conditions shown in Fig. 5(b), the material behaves similarly to Ga-rich conditions, with the $\mathrm{Ga}_{i}^{1+}$ dominant for electron chemical potentials through most of the band gap, but $V_{\mathrm{Ga} 3}^{2-}$ dominate for highly $n$-type conditions.

Analysis of the intragap levels introduced by the different defects, highlighted by the symbols in Fig. 5 that denote the defect charge state transitions, reveals a strong site- dependence of the electronic character of defects on Ga sites. In particular, the intragap levels introduced by $V_{\mathrm{Ga}}$ and $\mathrm{Te}_{\mathrm{Ga}}$ are significantly deeper on site 3 than site 1 . For the case of $V_{\mathrm{Ga}}$, which is an acceptor, the deep level corresponds to the transition from the -1 to -2 charge state, while for the negative $\mathrm{U} \mathrm{Te}_{\mathrm{Ga}}$ defects, the transitions are from +1 to -1 charge states. This site anisotropy is not observed for defects on Te sites $\left(V_{\mathrm{Te}}\right.$ and $\left.\mathrm{Ga}_{\mathrm{Te}}\right)$, for which the charge transition levels are not site-dependent. We will see later, however, that this site anisotropy on Ga sites does not carryover to the case of dopants and actually has much to do with electronic rearrangement at the Te sites surrounding the defects.

In Fig. 7, we show partial charge density plots for $V_{\mathrm{Ga} 1}$ and $V_{\mathrm{Ga} 3}$, to compare the electronic nature of the two $\mathrm{Ga}$ sites. Partial charge densities corresponding to the two highest fully occupied levels, a semi-filled state at the Fermi level, and the lowest fully unoccupied level are shown for each defect (see figure caption). The most striking differences occurs for the highest occupied level [(b) vs. (f)] and the semi-filled level [(c) vs. (g)]. These dissimilarities indicate a difference in the symmetry and the very nature of the levels associated with the same nominal defect $\left(e . g ., V_{\mathrm{Ga}}\right)$ when it is placed on the different Ga sites. More subtly, while the second highest occupied level [(a) vs. (e)] and the lowest unoccupied level [(d) vs. (h)] appear generally similar for the two sites, there is a more pronounced reorientation of the lone pairs on the surrounding Te atoms toward the defect for the $V_{\mathrm{Ga} 3}$ case compared to $V_{\mathrm{Ga} 1}$. This indicates that the lone pairs of the Te atoms around the defect reorient in order to partially reform the broken bonds left by the vacancy. This reorientation of the Te lone pairs does not occur for defects on Te sites, which are always surrounded by neighboring $\mathrm{Ga}$ atoms, explaining the lack of electronic anisotropy observed for defects on different Te sites.

To further understand the site anisotropy for defects on $\mathrm{Ga}$ sites, we examine in Fig. 8 the bonding configurations around Ga-site defects and the symmetry of the surrounding bond lengths. The lefthand-side figures correspond to defects on $\mathrm{Ga}$ site 1 , while the righthand-side figures correspond to defects on Ga site 3. Three different defects are shown for illustration: $V_{\mathrm{Ga}}, \mathrm{Te}_{\mathrm{Ga}}$, and $\mathrm{Ge}_{\mathrm{Ga}}$. The last defect, which is an impurity, is discussed in more detail in Sect. III C below.

Comparing the uppermost panels for the two $V_{\mathrm{Ga}}$ defects, we see why the reorientation of the Te lone pairs is more pronounced in rebuilding the broken bonds for $V_{\mathrm{Ga} 3}$ than for $V_{\mathrm{Ga} 1}$ : the distances between the remaining $\mathrm{Ga}$ atom near the vacancy and the surrounding Te atoms are smaller on average for $V_{\mathrm{Ga} 3}$ than they are for $V_{\mathrm{Ga} 1}$; the bond lengths are also more symmetric for $V_{\mathrm{Ga} 3}$. Moreover, we see that for $\mathrm{Te}_{\mathrm{Ga}}$ (the middle panels in Fig. 8) the symmetries of the atomic relaxations (JahnTeller distortions) are quite different on the two sites. This explains the change in the symmetry of the highest occupied and semi-filled levels on $V_{\mathrm{Ga}}$ illustrated in Fig. 7. Finally, in the bottom panels of Fig. 8, for the $\mathrm{Ge}_{\mathrm{Ga}}$ substitutional defect, we demonstrate that the distortions are much less pronounced for the case of substitutional impurities on $\mathrm{Ga}$, and the differences between sites 1 and 3 are correspondingly much less pronounced. (Here, we only show the Ge impurity, but this 

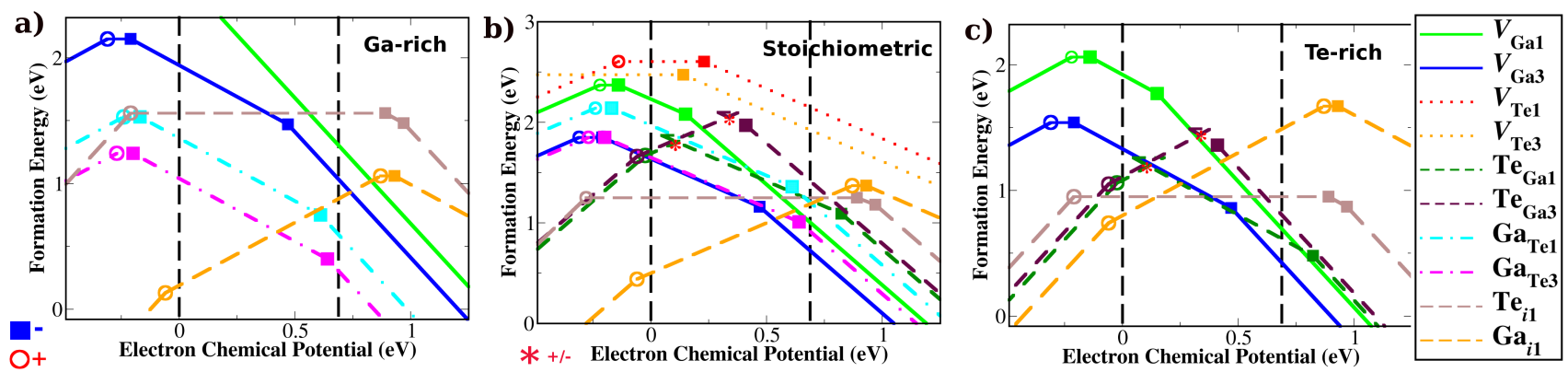

FIG. 5: (Color online) Formation energies of intrinsic defects in GaTe as a function of electron chemical potential, where the dashed vertical lines indicate the positions of the calculated valence band maximum (VBM) and conduction band minimum (CBM). Results for (a) Ga-rich, (b) stoichiometric, and (c) Te-rich are shown. The slope of each line segment indicates the charge state $q$ from Eq. (2) with the lowest formation energy for each defect over a given range of $\mu_{e}$. The symbols indicate the charge state crossings where a given defect changes charge. (The line segment continuations for each charge state above the lowest formation energy one are not shown for clarity.) Filled squares indicate acceptor levels (transitions involving capture of an electron or more negative charge), while open circles indicate donor levels (transitions involving release of an electron or more positive charge). Asterisks indicate negative-U transitions from $q=+1$ to $q=-1$ with no region of stability for the $q=0$ charge state. In (a) and (c), only the six lowest formation energy defects are shown, for clarity.
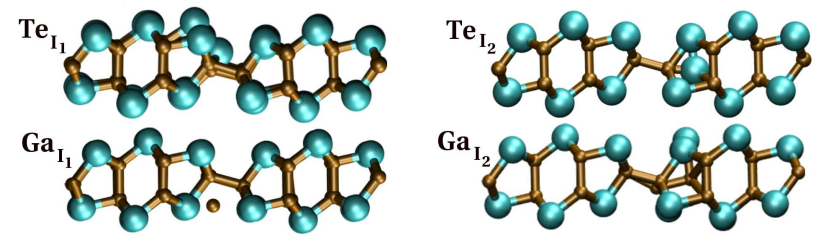

FIG. 6: (Color online) Relaxed structures of Te and Ga interstitials, comparing sites $I_{1}$ and $I_{2}$ from Fig. 3.

result is general for the set of impurities we have studied and discuss later in Sect. III C.) As we will show in more detail in Sect. III C, substitutional impurities on Ga, like all defects studied on the different Te sites, do not show significant site anisotropy of their electronic structures.

\section{Intragap levels: theory vs. experiments}

Experimentally, at least three deep acceptor states (at 0.40, 0.59 , and $0.67 \mathrm{eV}$ above the VBM) and one shallow acceptor level (at $0.14 \mathrm{eV}$ above the VBM) were observed in undoped GaTe using deep level transient spectroscopy (DLTS) and low-temperature photoluminescence, respectively. ${ }^{6,8}$ The shallow level was tentatively assigned to the $0 /-$ transition of $V_{\mathrm{Ga}}$, while attempts to associate the deep levels to specific intrinsic defects were made based on the thermal history behavior of DLTS peaks, particularly whether the peaks are quenched, enhanced, or stable to thermal annealing. Thus, in Ref. 6, tentative assignment was made of the $0.67 \mathrm{eV}$ deep level to doubly ionized $V_{\mathrm{Ga}}$, since the peak was thermally enhanced; the $0.59 \mathrm{eV}$ peak top $\mathrm{Te}_{\mathrm{Ga}}$, since the peak was thermally stable; and the $0.40 \mathrm{eV}$ peak to a complex between $\mathrm{Ga}$ interstitial and $\mathrm{Ga}$ vacancy $\mathrm{Ga}_{i}-V_{\mathrm{Ga}}$, since the peak was thermally quenched.

In Fig. 9, we show the calculated acceptor levels (red lines) of all intrinsic defects overlaid on the experimentally observed levels (blue dotted lines). We see that indeed Ga vacancies introduce very shallow acceptor levels. However, $\mathrm{Ga}_{\mathrm{Te}}$ antisites also introduce very shallow levels and should be much more prevalent than vacancies under Ga-rich conditions due to the lower formation energies [see Fig. 5(a)]. The $\mathrm{Ga}_{\mathrm{Te}}$ double ionization could also be consistent with the higher energy deep levels observed experimentally. $\mathrm{Te}_{\mathrm{Ga}}$ antisites also show reasonable, but not unambiguous, alignment with the measured data. A particular question is whether all four of the predicted levels (two each for each Ga site) are separately observable and consistent with the data.

Since the concentrations of both $\mathrm{Ga}$ and Te interstitials are significant under all growth conditions, one might argue that for Te-rich material, annealing drives $\mathrm{Te}_{i}$ to occupy $V_{\mathrm{Ga}}$ sites, creating $\mathrm{Te}_{\mathrm{Ga}}$ antisites and enhancing charge trapping by these antisites. On the other hand, one could argue that under Garich conditions, thermal treatment drives the reaction of $\mathrm{Ga}_{\mathrm{Te}}$ with $\mathrm{Te}_{i}$ to form $\mathrm{Ga}_{i}$, that could then occupy $\mathrm{Ga}$ vacancies, quenching charge trapping associated with both $\mathrm{Ga}$ vacancies and antisites. Thus, it is difficult to assign levels to specific defects based solely on the thermal response. It is particularly important to know the stoichiometric regime of the crystal during growth and/or annealing. Additionally, as shown above, one must consider the possibility that the electronic anisotropy of defects on different Ga sites, such as $V_{\mathrm{Ga}}$ and $\mathrm{Te}_{\mathrm{Ga}}$, can result in two distinct levels observed for the same nominal defect on different sites. Figure 9 clearly shows how the theoretical results for both $V_{\mathrm{Ga}}$ and $\mathrm{Te}_{\mathrm{Ga}}$ can be consistent with the experimental data when accounting for the site anisotropy of the electronic behavior of these defects.

Given the experimental observation of excess Te atoms at grain boundaries in the crystals and reports of $p$-type conductivity $^{4-9}$ (see below), we believe that GaTe is grown mostly in a Te-rich environment. Then, it is reasonable from our theoretical results to assign the measured deep acceptors to the doubly ionized Ga vacancy and Te ansitites, as proposed, since these defects introduce deep levels consistent with the experimental data and also are dominant defects in 
a)

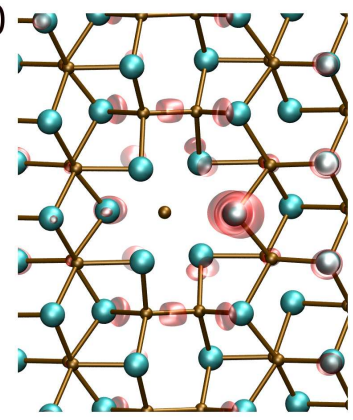

e)

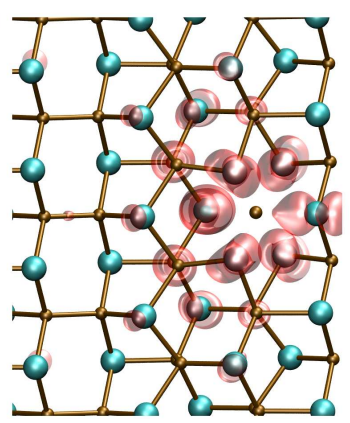

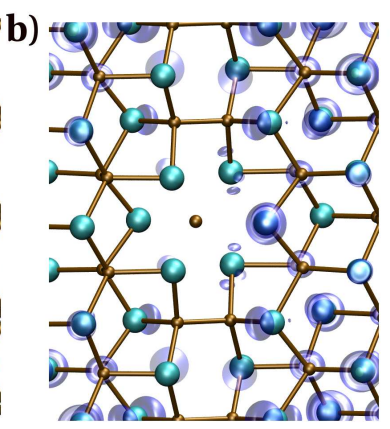

f)

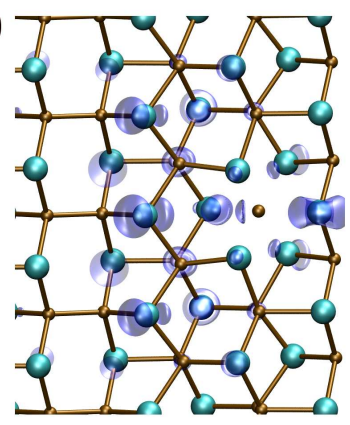

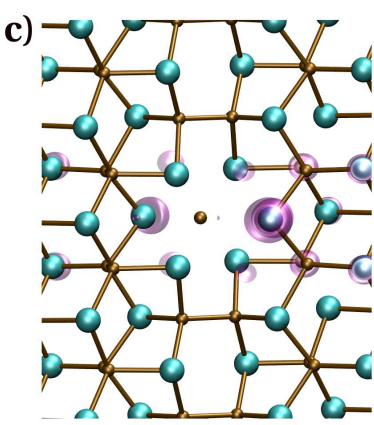

g)

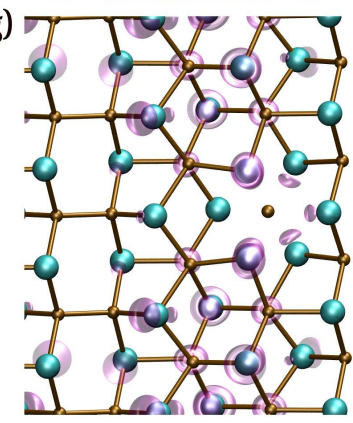

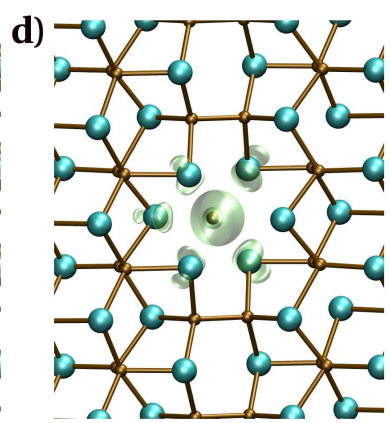

h)

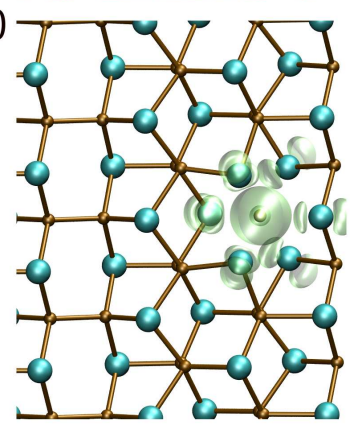

FIG. 7: (Color online) Partial charge densities of the $V_{\mathrm{Ga} 1}(\mathrm{a}-\mathrm{d})$ and $V_{\mathrm{Ga} 3}(\mathrm{e}-\mathrm{f})$ defects, for states near the Fermi level. The states shown in (a), (b), (e), and (f) are the highest fully occupied levels; (c) and (g) are semi-filled states at the Fermi level; and (d) and (h) are the lowest fully unoccupied levels. The energy of the states increases from left to right in the figure. Comparison of (b) and (f), as well as (c) and (g), shows the markedly different nature of the molecular orbitals on the two Ga sites. The isosurface in each plot encloses half of the total charge in the corresponding level.

equilibrium under Te-rich conditions. The shallow level is consistent with single ionized $V_{\mathrm{Ga}}$. However, tests we performed for defect complexes consisting of a Ga vacancy (on site 1) and a neighboring Te interstitial (on two possible sites $I_{1}$ and $I_{2}$ ) resulted in relaxation to $\mathrm{Te}_{\mathrm{Ga}}$, indicating that the vacancy-interstitial complex is not stable. The $V_{\mathrm{Ga}}-\mathrm{Ga}_{i}$ complex is similarly unstable, particularly considering that the isolated defects $V_{\mathrm{Ga}}$ and $\mathrm{Ga}_{i}$ are oppositely charged (see Fig. 5) which leads to a Coulombic attraction and annihilation of the defects rather than complex formation.

We note that the interpretation of the defect formation energy calculations above, in light of computing equilibrium carrier concentrations, is sensitive to the band gap underestimate of DFT. In our analysis, we do not employ any ad hoc band gap correction scheme, but rather interpret the formation energies within the calculated band edges indicated in Fig. 5. Various schemes have been employed in the literature, including a rigid shift of the conduction band edge to match the experimental band gap, ${ }^{30-34}$ however recent work combining DFT and $G_{0} W_{0}$ calculations showed that such ad hoc schemes applied without proper justification can lead to poorer prediction of defect concentrations than application of no correction at all. $^{11}$

Our results for the pristine crystal suggest that growing GaTe in Ga-rich conditions could suppress at least one of the near-midgap deep levels by substituting $\mathrm{Te}_{\mathrm{Ga}}$ (which introduces one shallow and one deep level) for $\mathrm{Ga}_{\mathrm{Te}}$ (which introduces two deep levels). Reducing the concentration of such deep levels would contribute to increased carrier lifetimes in the material. In addition, Ga-rich conditions reduce the occurrence of $V_{\mathrm{Ga}}$ shallow acceptors in favor of $\mathrm{Ga}_{i}$ shallow donors, promoting better carrier compensation (see Section III E) and higher material resistivity than presently observed in as-grown $p$-type material. Finally, we shall show in section III F that $\mathrm{Ga}_{i}$ and $\mathrm{Ga}_{\mathrm{Te}}$ are much weaker scatterers than $\mathrm{Te}_{i}$ and $\mathrm{Te}_{\mathrm{Ga}}$.

\section{B. Accidental impurities}

As mentioned above, as-grown GaTe has always been reported to posses $p$-type character. However, an analysis of the net carrier concentrations in intrinsic material (discussed in more detail below in Sect. IIIE), based on the intrinsic defect formation energies calculated above, predicts $n$-type intrinsic material except for the most Te-rich conditions [see Fig. 13(a)]. The $n$-type character of Ga-rich intrinsic material arises from a large concentration of $\mathrm{Ga}_{i}$, while there is a more complicated interplay of defects for extreme Te-rich conditions. Thus, accidental impurities in the as-grown material may be significant, particularly in regard to creating the observed $p$-type conductivity.

Here, we consider $\mathrm{O}$ and $\mathrm{H}$ as possible accidental impurities. Figure 10 shows the calculated formation energies for $\mathrm{O}$ and $\mathrm{H}$ incorporated on various substitutional and interstitial sites, for Te-rich conditions. For O [Fig. 10(a)], the behavior is similar to Te intrinsic defects, both elements being group VI chalcogens. The dominant defects are $\mathrm{O}$ interstitials, however they are electronically neutral throughout the band gap. 

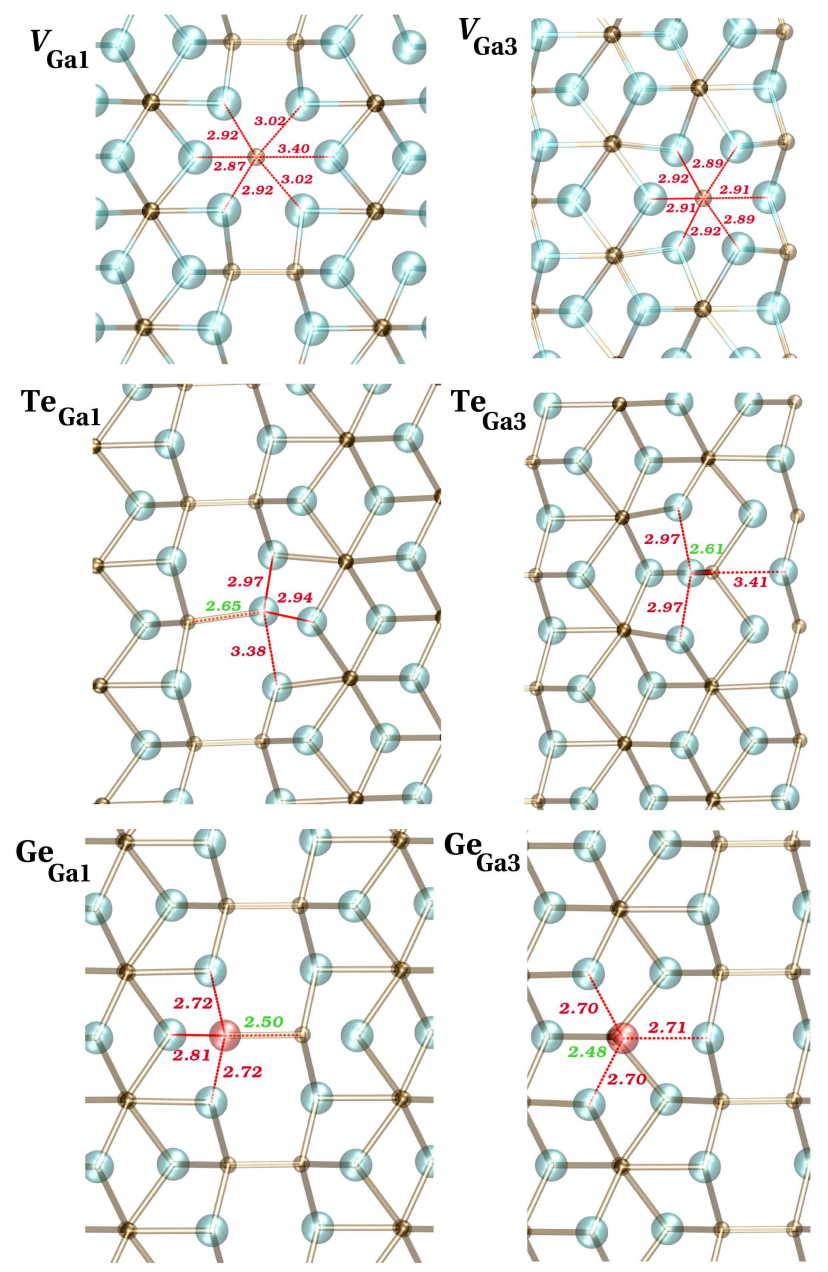

FIG. 8: (Color online) Relaxed symmetries and distances between atoms around defects on different $\mathrm{Ga}$ sites. The $V_{\mathrm{Ga}}, \mathrm{Te}_{\mathrm{Ga}}$, and $\mathrm{Ge}_{\mathrm{Ga}}$ defects are compared for Ga sites 1 and 3. Jahn-Teller distortions are much more pronounced for $V_{\mathrm{Ga}}$ (top panels) and $\mathrm{Te}_{\mathrm{Ga}}$ (middle panels) than for a substitutional Ge dopant (bottom panels).

Substitutional $\mathrm{O}_{\mathrm{Te}}$ is also neutral, while $\mathrm{O}_{\mathrm{Ga}}$ introduces a deep acceptor state, but the formation energy of Ga substitutions is considerable higher than for the other $\mathrm{O}$ defects.

Hydrogen defects [Fig. 10(b)] are all shallow acceptors, with interstitial incorporation again favored. Substitutional $\mathrm{H}_{\mathrm{Ga}}$ is a shallow double acceptor which is dominant for Fermi levels close to the conduction band minimum, while $\mathrm{H}_{\mathrm{Te}}$ substitutions are not favored. The strong acceptor nature of $\mathrm{H}$ impurities in GaTe implies that growth processes that inhibit $\mathrm{H}$ incorporation may improve material resistivity (more details in Sect. IIIE). Oxygen impurities, on the other hand, while highly soluble in the material, may not be too detrimental from a resistivity/free-carrier point of view.

\section{Dopants}

Judicious doping of the semiconductor can be an effective means to optimize the resistivity, carrier mobility, and carrier

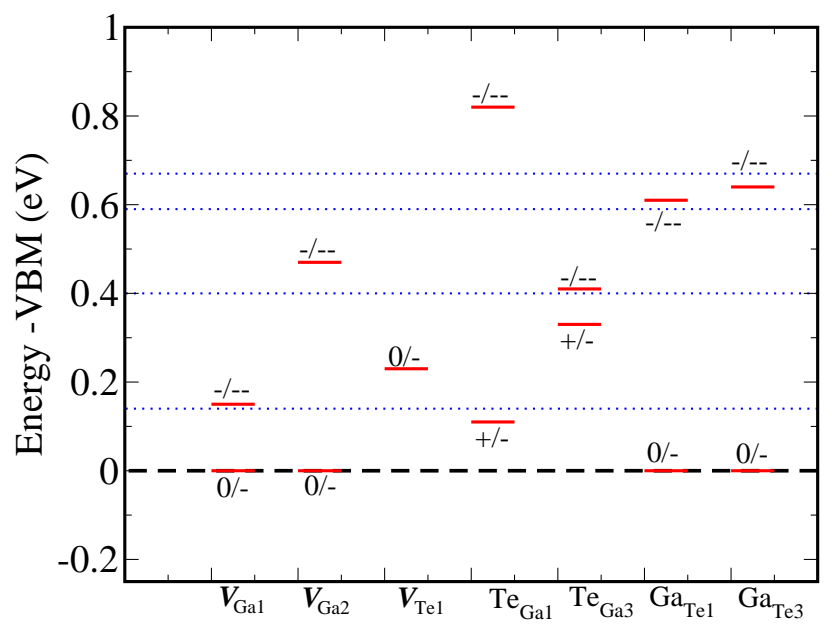

FIG. 9: (Color online) Acceptor levels for all intrinsic defects in $\mathrm{GaTe}$ relative to the valence band maximum (set to $0 \mathrm{eV}$ ). The red lines indicate the positions of charge state transitions of each defect, which were marked by squares in Fig. 5 . The blue dotted lines indicate the positions of experimentally measured levels. ${ }^{6}$
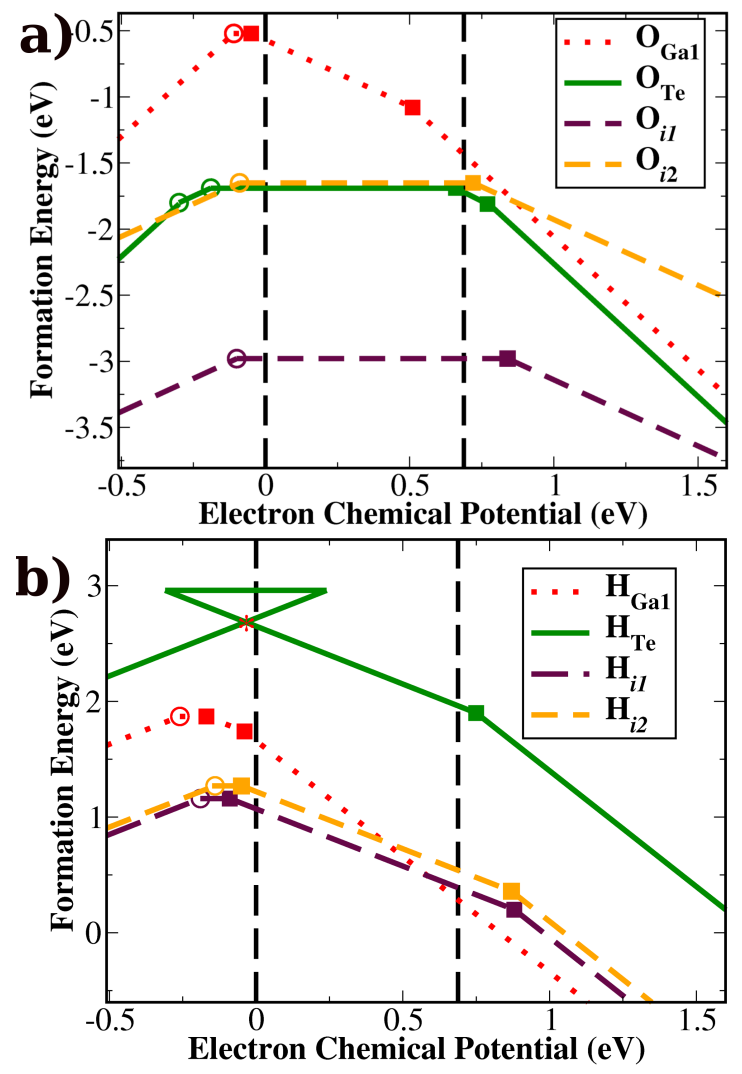

FIG. 10: (Color online) Formation energies of (a) oxygen and (b) hydrogen impurities on various incorporation sites in $\mathrm{GaTe}$, for Terich conditions. Calculated LDA band gap is indicated by the vertical dashed lines. 


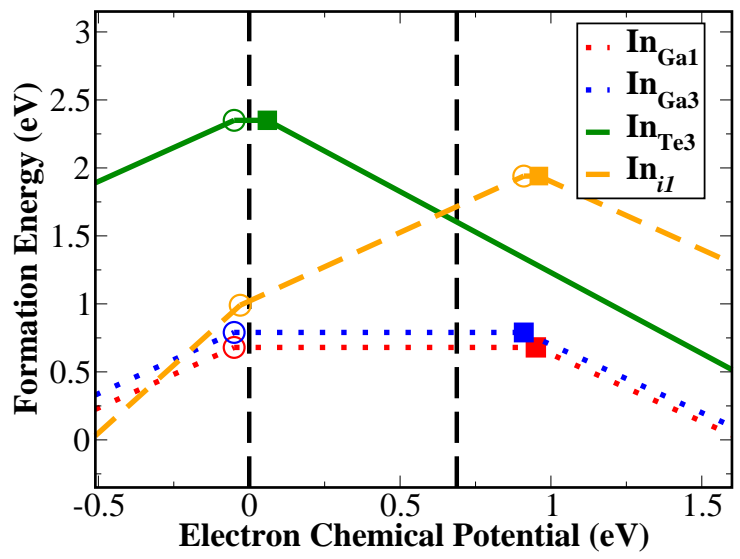

FIG. 11: (Color online) Formation energies of In dopants in GaTe, incorporated on different sites, for Ga-rich conditions. Calculated LDA band gap is indicated by the vertical dashed lines.

lifetime. This can be achieved by introducing Fermi-level pinning and/or carrier compensating defects and by modifying the presence of carrier trapping deep centers. ${ }^{12,35,36}$ For GaTe, which has been shown experimentally to present an excess of holes, we seek a dopant that will compensate the material with free electrons. Thus, natural candidates are elements that substitute the cations with higher valency atoms, such as group IV or $\mathrm{V}$ elements substituting $\mathrm{Ga}$. Isovalent doping may also be advantageous if certain electronic property modification arise due mainly to differences in electronegativity of the dopant atom compared to the host atom.

Experimentally, isovalent doping of GaTe with In was attempted. ${ }^{6}$ It was observed that one of the deep levels identified in the as-grown material disappeared and a new shallow level appeared. The authors argued that In atoms could occupy Ga vacancies, which might suppress the occurrence of both deep and shallow levels related to $V_{\mathrm{Ga}}$ and introduce a new shallow level. The new shallow level was speculated to be a complex of $\operatorname{In}_{i}-V_{\mathrm{Ga}}$.

We tested computationally the possibilities of doping GaTe both with isovalent and higher valency elements. The results for In and Ge are shown in Figs. 11 and 12, respectively.

We find that In prefers to incorporate by substitution of Ga sites (both sites 1 and 3 about equally), with $\operatorname{In}_{\mathrm{Ga}}$ being electronically inactive (neutral throughout the band gap). Interstitial In is a shallow donor, and the lowest formation energy electrically active defect. Since the fraction of In dopants incorporated as interstitials is expected to be low $\left(\propto \exp \left[-\left(\Delta E_{f, \mathrm{In}_{i}}-\Delta E_{f, \mathrm{In}_{\mathrm{Ga}}}\right) / k_{B} T\right]\right.$ at equilibrium), indium doping may be able to contribute to compensation of the intrinsic acceptor defects, but would also contribute a much larger concentration of neutral substitutional defects. Substitutional $\mathrm{In}_{\mathrm{Te}}$ are shallow acceptors, but these are not expected to contribute due to the much higher formation energy. In addition to the formation energies for the Ga substitutions not showing a site dependence, Fig. 11 shows that the electronic properties also are not site dependent for In dopants. This lack of Ga site dependence seems to be universal for extrinsic defects (see also Fig. 12, for example), which is counter to the
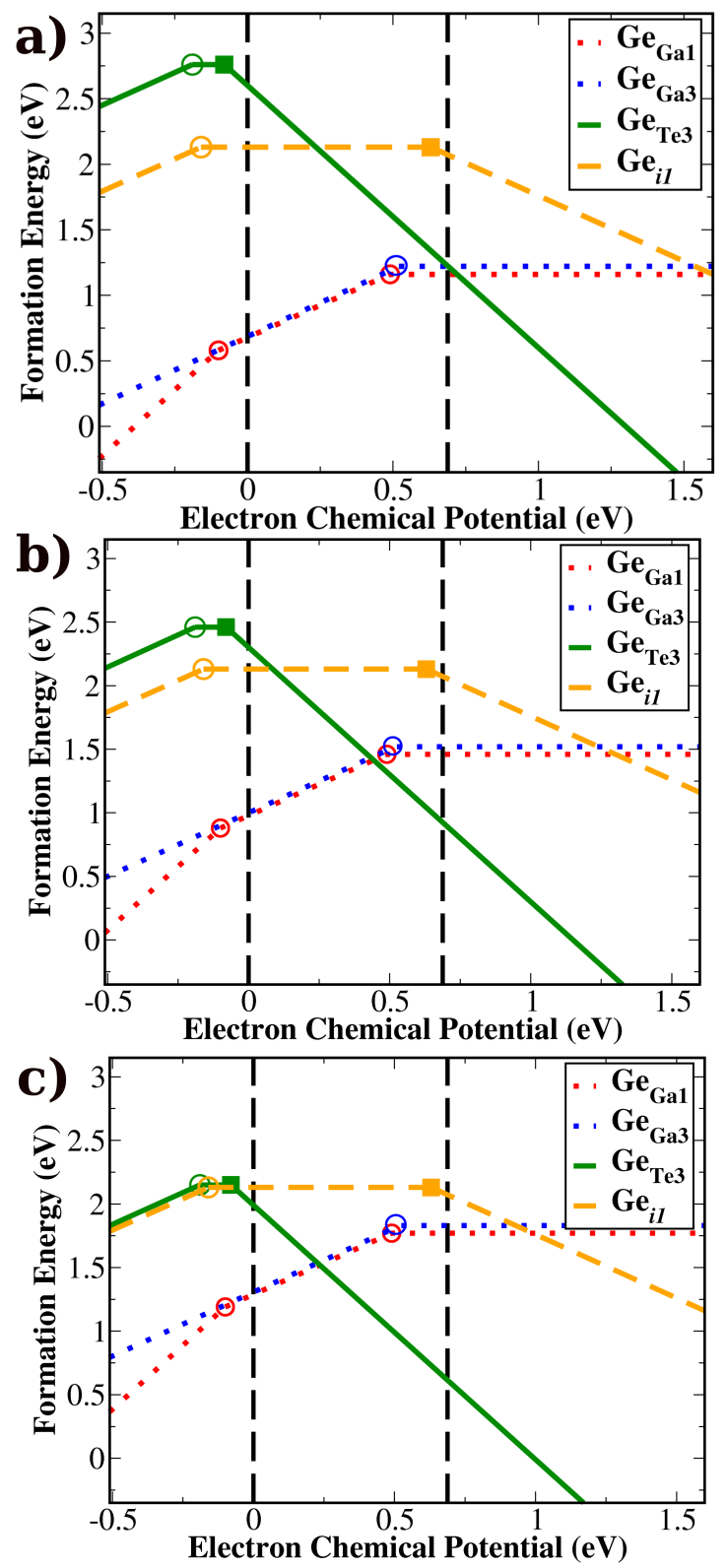

FIG. 12: (Color online) Formation energies of Ge dopants in GaTe, incorporated on different sites, for (a) Te-rich, (b) stoichiometric, and (c) Ga-rich conditions. Calculated LDA band gap is indicated by the vertical dashed lines.

behavior of intrinsic defects like $\mathrm{Te}_{\mathrm{Ga}}$ or $V_{\mathrm{Ga}}$. Our calculations for the $\operatorname{In}_{i}-V_{\mathrm{Ga}}$ complex predicted it to be unstable, due to the Coulombic attraction between the oppositely charged isolated defects leading to annihilation into the neutral $\mathrm{In}_{\mathrm{Ga}}$; thus, no new shallow level is predicted to occur from such a complex.

Germanium, as a group IV element, is a donor when substituting $\mathrm{Ga}$, which is favored for Te-rich conditions [see Fig. 12(a)]. In Ref. 25, theoretical calculations of germanium on gallium sites in GaTe were reported with results similar to owers. The authors, however, did not consider Te sites for Ge incorporation. Again, we see no Ga site dependency. For Ga-rich conditions, $\mathrm{Ge}_{\mathrm{Te}}$ double shallow acceptors are at least 
comparable in concentration to $\mathrm{Ge}_{\mathrm{Ga}}$. Interstitial $\mathrm{Ge}$ is not favored under any conditions and is either a neutral defect or possibly introduces a very deep acceptor state. At some value of chemical potential difference between stoichiometric and the extreme Ga-rich limit, the formation energies for $\mathrm{Ge}_{\mathrm{Ga}}^{1+}$ and $\mathrm{Ge}_{\mathrm{Te}}^{2-}$ cross, leading to an interesting site-dependent ambipolar behavior. Such behavior may be exploited to achieve self-compensation through a careful annealing process. For example, by growing the material under Ga-rich conditions then partially annealing for a time in a Te-rich environment, a controlled conversion of a fraction of initially incorporated $\mathrm{Ge}_{\mathrm{Ga}}$ dopants into $\mathrm{Ge}_{\mathrm{Te}}$ and a consequent pinning the Fermi level could be achieved. A similar type of ambipolar behavior was recently observed for Sn dopants in AlSb. ${ }^{12}$

Indeed, GaTe samples doped mildly with Ge experimentally showed better response to radiation measurements. ${ }^{37} \mathrm{Im}$ proved control of the Ge incorporation could further improve the resistivity, as our more quantitative analysis in Sect. III E shows. Interestingly, our results here related to Ge co-doping support the conclusion from Sect. III A on intrinsic defects, that Ga-rich growth should promote higher quality radiation detection material.

Further discussions of the effects of In and Ge dopants on the net carrier concentration (resistivity) and carrier scattering (mobility) are found below in Sects. IIIE and IIIF.

\section{Competing phases}

When atoms of extra elements are added to the growth process with the intention of doping the resulting material, in addition to simply becoming incorporated into the lattice of the pristine material, there is the possibility that such dopants will form competing phases with one or more of the original elements. For example, In can react with $\mathrm{Ga}$ and/or $\mathrm{Te}$ atoms to form InTe, $\mathrm{In}_{2} \mathrm{Te}_{3}, \mathrm{In}_{2} \mathrm{Te}_{5}, \mathrm{In}_{4} \mathrm{Te}_{3}, \mathrm{GaInTe}_{2}$, and other compounds. As a matter of fact, it has been shown that the formation of InTe may be crucial to obtain high quality ohmic contacts on GaTe, whereas the formation of $\mathrm{GaInTe}_{2}$ is detrimental. ${ }^{38}$ Germanium also combines with Te to form GeTe. In addition to these phases, Ga and Te can also combine with $\mathrm{O}$ to form a very complex structure. ${ }^{39}$ The presence of possible competing phases restricts the range of chemical potentials in Eq. (2). This issue is partially approached in 30 . Qualitatively, the formation of competing phases can have the effect of removing an excess of a certain host element in the crystal as it reacts to form the secondary phase. For example, since In and Ge combine with Te atoms rather than gallium to form compounds, the presence of In or Ge in GaTe can help to shift the chemical equilibrium towards Ga-rich/Te-poor conditions. As already noted above, Ga-rich growth conditions should promote higher quality radiation detection material, so In and/or Ge doping may be favorable in this regard. This chemical effect is in addition to the doping effect when the element is incorporated into the host crystal lattice, modifying the net carrier concentration and shifting the electron chemical potential. These latter effects are discussed in the next section.

\section{E. Net carrier concentration}

Using the results from the previous sections, we can apply Eq. (5) for the charged defects to calculate the concentration of free carriers - electrons and holes - introduced in the GaTe crystal by intrinsic and extrinsic defects. The prefactor $c_{0}$ in Eq. (5) corresponds to the density of possible incorporation sites for the specific defect, including a multiplicity factor that accounts for symmetry. All atomic sites in the primitive cell of GaTe are on Wyckoff positions "i" (of group C $2 / \mathrm{m}$ unique axis b). In our calculations, we have grouped together sites labeled 2 and 3 on Fig. 3, since the atomic environments and electronic properties are very similar for those two sites for both $\mathrm{Ga}$ and Te atoms, as indicated by the PDOS analysis shown in Fig. 4. Therefore, for defects on sites 2 and 3, the prefactor $c_{0}=\frac{2}{3} \Omega_{0}^{-1}$, where $\Omega_{0}$ is the primitive unit cell volume), whereas for defects on site $1, c_{0}=\frac{1}{3} \Omega_{0}^{-1}$. The multiplicities of interstitial sites also follow this rule, since even defects having starting configurations with lower multiplicities than Wyckoff position "i" (such as the $I_{1}$ site on Wyckoff position "b") were found to relax into positions "f," "g," or "h," which have the same multiplicity as position "i."

Charged defects will introduce either holes or electrons in the crystal depending on whether they are acceptors $(q<0)$ or donors $(q>0)$, respectively. Those free carriers are balanced by the total of charged defect cores left behind through the charge neutrality equation

$$
n\left(\mu_{e}\right)+\sum_{i: q_{i}<0} q_{i} c_{i}=p\left(\mu_{e}\right)+\sum_{j: q_{j}>0} q_{j} c_{j},
$$

where the $c_{i}$ are the concentrations of charged defects given by Eq. (5), $q_{i}$ are the charges, and $n\left(\mu_{e}\right)$ and $p\left(\mu_{e}\right)$ are the concentrations of free electrons and free holes in the material, respectively, given by

$$
\begin{array}{r}
n\left(\mu_{e}\right)=\int_{C B M}^{\infty} D(\varepsilon) \cdot\left[1+\exp \left(\frac{\varepsilon-\mu_{e}}{k_{b} T}\right)\right]^{-1} d \varepsilon \\
p\left(\mu_{e}\right)=\int_{\infty}^{V B M} D(\varepsilon) \cdot\left\{1-\left[1+\exp \left(\frac{\varepsilon-\mu_{e}}{k_{b} T}\right)\right]^{-1}\right\} d \varepsilon,
\end{array}
$$

where $D(\varepsilon)$ is the electronic density of states of the host crystal. In Eq. (6), the summation on the left hand side represents a sum over all acceptors and on the right hand side a sum over all donors in the crystal. Note that the formation energy given by Eq. (2) that appears in Eq. (5) for $c_{i}$ depends on the electron chemical potential $\mu_{e}$. However, $\mu_{e}$ is modified by introducing free carriers in the material. Therefore, to obtain the equilibrium electronic chemical potential $\mu_{e}$, which is a priori unknown, we need to solve Eqs. (5), (6), and (7) iteratively to obtain $\mu_{e}, n$, and $p$ self-consistently.

When evaluating these equations in the presence of extrinsic defects, we fix the total concentration of each extrinsic defect in the crystal and obtain the relative fraction in each possible incorporation site and charge state from the formation energies. This means that we do not treat the calculated formation energies of extrinsic defects as absolute values that are directly compared to the formation energies of the intrinsic defects. Note that the equilibrium temperature for defect 
formation in Eq. (5) and the free carrier temperature in Eq. (7) should be independently chosen. The latter corresponds to the actual operating condition of the device (taken as $300 \mathrm{~K}$ in this work) since the free carriers will thermalize; the former is associated with freeze-in of defects as the material cools down from the melt and defect diffusivities decrease. In our analysis, we assume a constant freeze-in temperature for all defects, which we treat as a parameter set to an elevated temperature in the range of $600 \mathrm{~K}$ up to the growth (melt) temperature of $1100 \mathrm{~K}(1097 \mathrm{~K})$.

In Fig. 13(a) we show the calculated concentration of net carriers in intrinsic GaTe as a function of the chemical potential difference $\Delta \mu=\mu_{T e}-\mu_{G a}$ (negative $\Delta \mu$ is Garich/Te-poor and positive $\Delta \mu$ is Te-rich/Ga-poor). The solid lines indicate excess electrons ( $n$-type conductivity), while the dashed lines indicate excess holes ( $p$-type), so we see that a type change occurs at moderately Te-rich conditions. For most conditions, from Ga-rich to moderately Te-rich, the intrinsic material is $n$-type; only under highly Te-rich conditions is the intrinsic material predicted to be $p$-type. This behavior is a consequence of the high occurrence of $\mathrm{Ga}_{i}$, which is the only donor of all intrinsic defects studied. The occurrence of $\mathrm{Ga}_{i}$ is significant even for Ga-poor conditions, but for highly Te-rich conditions, other defects such as $V_{\mathrm{Ga}}$ and $\mathrm{Te}_{\mathrm{Ga}}$ (both acceptors) are more prevalent and switch the material $p$-type. As-grown material has always been reported to show $p$-type conductivity experimentally, ${ }^{4-9}$ which hints that unintentional impurities convert the intrinsic material to $p$-type conductivity. We note generally a moderately low intrinsic net free carrier concentration overall, if no impurities are present.

Therefore, to explain the observed $p$-type character of GaTe, we either must have material grown under very Terich conditions or we have to consider accidental incorporation of impurities. For this purpose, we consider two of the most ubiquitous contaminants, $\mathrm{O}$ and $\mathrm{H}$, as already discussed in Sect. III B. In Figs. 13(b) and (c) we show the net carrier density of intrinsic GaTe including an added impurity concentration of $10^{17} \mathrm{~cm}^{-3}$ of $\mathrm{O}$ or $\mathrm{H}$, respectively. We see that O impurities [Fig. 13(b)] do not have a large impact on the free carrier concentration of GaTe, since most $\mathrm{O}$ impurities are neutral as shown above in Fig. 10(a). Hydrogen, on the other hand, dramatically changes the situation, since those impurities add acceptors to the material [see Fig. 10(b)] and make it p-type under most conditions. For illustration, in Fig. 13(d) we show the result of including both $\mathrm{O}$ and $\mathrm{H}$ impurities, but with the concentration of $\mathrm{O}$ impurities set two orders of magnitude higher $\left(10^{19} \mathrm{~cm}^{-3}\right)$ than $\mathrm{H}\left(10^{17} \mathrm{~cm}^{-3}\right)$. Even with the concentration of $\mathrm{O}$ impurities being two orders of magnitude above that of $\mathrm{H}$, we still obtain $p$-type character for most conditions, in line with the experimental reports. Since these impurities are prevalent, their presence likely represents the state of as-grown undoped experimental material. As a model for the as-grown undoped material, we take the conditions of Fig. 13(d).

In Fig. 14 we analyze the compensation doping effects of Ge and In, as discussed above in Sect. IIIC. We begin with the baseline as-grown material from Fig. 13(d) (including $10^{17} \mathrm{~cm}^{-3} \mathrm{H}$ and $10^{19} \mathrm{~cm}^{-3} \mathrm{O}$ ) and add increasing con- centrations of Ge: $5 \times 10^{16}, 10^{17}$, and $5 \times 10^{17} \mathrm{~cm}^{-3}$, for Figs. 14(a), (b), and (c), respectively. Although fairly good compensation is seen in Fig. 14(b), it is also evident from Figs. 14(a) and (c) that small variations of only half an order of magnitude in the concentration of Ge completely change the type of dominant free carrier in the material and the compensation is correspondingly very sensitive. Therefore, direct compensation of GaTe with $\mathrm{Ge}$, although possible, needs to be very carefully tuned. We have already discussed in Sect. III C that Ge can act as either a donor or an acceptor, depending on whether incorporated on Ga or Te sites, both of which are favorable under different chemical environments. Thus, a careful annealing process with the chemical environment held at the opposite extreme from the growth environment, as described above in Sect. III C, may be used to tune the compensation for a given incorporated concentration of Ge. This process would correspond to tuning horizontally along the curves in Fig. 14 to find the type-crossover point or minimum carrier concentration point.

Finally, Fig. 14(d) shows the result for the same situation as in Fig. 14(b) (H: $10^{17} \mathrm{~cm}^{-3}$, O: $10^{19} \mathrm{~cm}^{-3}$, Ge: $10^{17} \mathrm{~cm}^{-3}$ ) but also adding $10^{19} \mathrm{~cm}-3$ In dopants. It is clear that the addition of In, even at such high concentrations, does not affect very much the density of free carriers in the material. This is expected, since Fig. 11 shows that In is electronically inactive on Ga sites, introducing electrons only when it is present on interstitial or Te sites, which are not favorable due to the higher formation energy. However, as discussed in Sect. III D, the presence of In can shift the chemical equilibrium towards Ga-rich conditions during growth, which reduces the occurrence of $V_{\mathrm{Ga}}$, affecting the compensation and net carrier concentration. Thus, the results here are not incompatible with the experimental observation that incorporation of In in GaTe increases the resistivity of the material. ${ }^{6}$

\section{F. Carrier scattering by defects}

In addition to possibly introducing free carriers that add background noise to a radiation detector and possibly introducing intragap levels that trap carriers, defects also can reduce the mobility of photon-induced carriers by disturbing the otherwise perfectly periodic lattice and introducing scattering centers. Here, we are mainly concerned with comparing and ranking the detrimental effect on carrier mobility of a set of defects (intrinsic or impurity), related to the lattice distortion introduced by the defect. The additional effect of Coulomb scattering degrades the carrier mobility as well (for charged defects), however this long-range effect is essentially identical for all defects with the same magnitude of charge. Since most of the pertinent defects for this analysis are either single charged or neutral, we simply focus on the relative scattering rates due to the lattice distortion and assume a constant Coulomb scattering contribution for charged defects.

To calculate the carrier scattering rate by a defect, we use Fermi's golden rule to evaluate the scattering rate between two electronic states $\Psi_{f}\left(k^{\prime}\right)$ and $\Psi_{i}(k)$, with energies $\varepsilon_{f}$ and $\varepsilon_{i}$, 

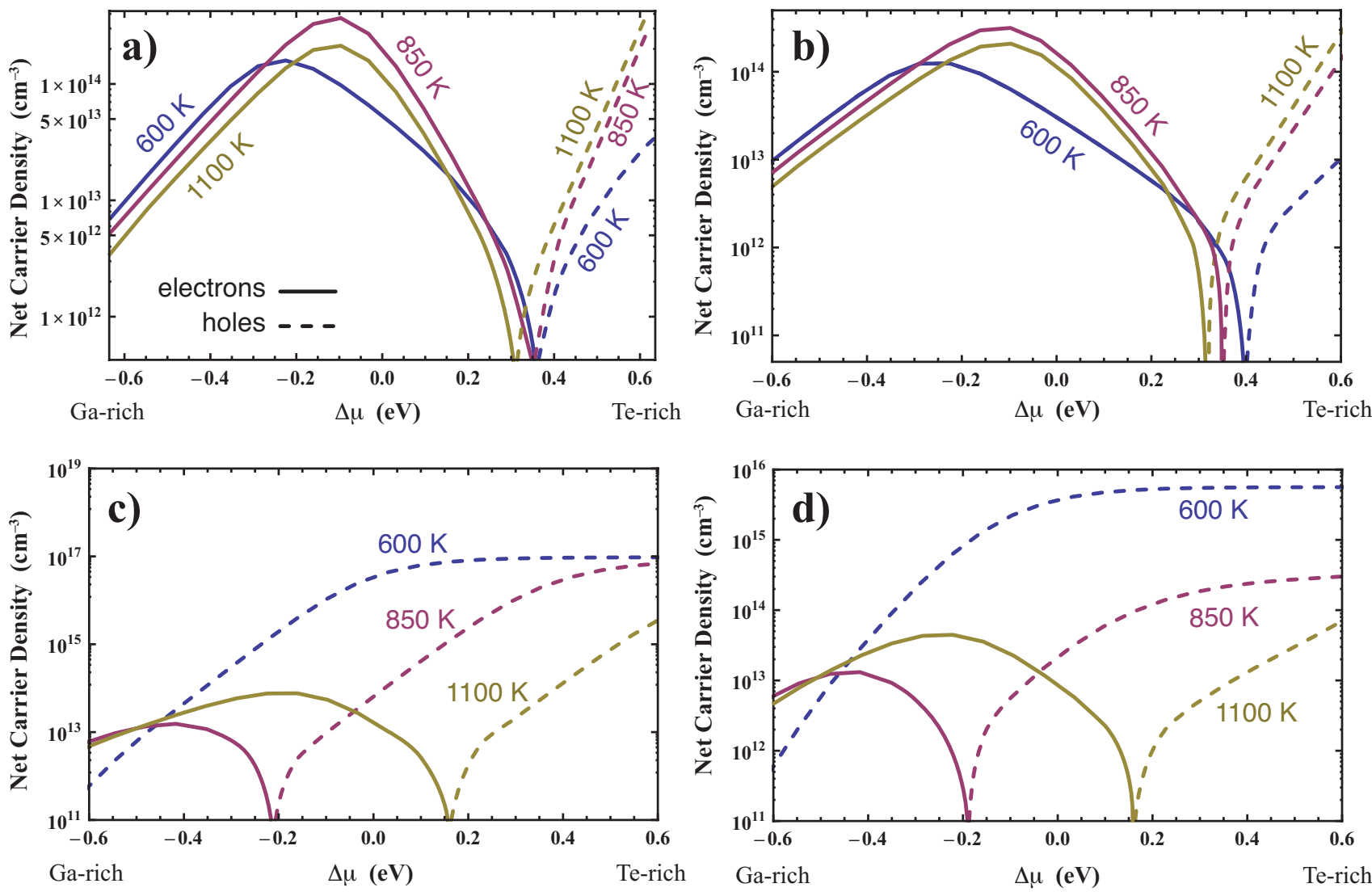

FIG. 13: (Color online) Net carrier concentration at $300 \mathrm{~K}$ as a function of chemical potential difference $\Delta \mu=\mu_{T e}-\mu_{G a}$, for different defect freeze-in temperatures indicated on the plots (blue lines: $600 \mathrm{~K}$, magenta lines: $850 \mathrm{~K}$, yellow lines: $1100 \mathrm{~K}$ ). (a) Intrinsic defects only; (b) intrinsic defects $+10^{17} \mathrm{~cm}^{-3} \mathrm{O}$ impurities; (c) intrinsic defects $+10^{17} \mathrm{~cm}^{-3} \mathrm{H}$ impurities; (d) intrinsic defects $+10^{19} \mathrm{~cm}^{-3} \mathrm{O}+10^{17} \mathrm{~cm}^{-3}$ $\mathrm{H}$ impurities. Thick lines indicate excess electrons ( $n$-type conductivity), while thin lines indicate excess holes ( $p$-type conductivity). Positive $\Delta \mu$ corresponds to Te-rich conditions, while negative $\Delta \mu$ corresponds to Ga-rich.

as $^{10}$

$$
W_{i j}=\frac{2 \pi}{\hbar}\left|\left\langle\Psi_{f}\left(k^{\prime}\right)\left|V_{\text {pert }}\right| \Psi_{i}(k)\right\rangle\right|^{2} \delta\left(\varepsilon_{f}-\varepsilon_{i}\right)
$$

where $V_{\text {pert }}(\mathbf{r})=V_{d}(\mathbf{r})-V_{0}(\mathbf{r})$ is the perturbation potential taken as the difference of the total potential in the defect cell, $V_{d}(\mathbf{r})$, and the ideal cell, $V_{0}(\mathbf{r})$, with the defect cell including full atomic relaxation. Equation 8 must be averaged over all pairs of states in the Brillouin zone to get the total scattering rate, but it has been shown that a relative measure of the average scattering rate can be obtained from $V_{\text {pert }}$ alone via ${ }^{10}$

$$
\tilde{M}^{2}=\left(\int d \mathbf{r}\left|\nabla V_{\text {pert }}\right|\right)^{2}
$$

Figure 15 shows the results of evaluating Eq. (9) for the set of defects considered in this work for GaTe. For both $\mathrm{Ga}$ and Te interstitials, only site $I_{1}$, the one with the lowest formation energy among the four tested, is included. We see that $\mathrm{Te}_{i}$ introduce the highest scattering among intrinsic defects, followed by $V_{\mathrm{Ga}}$ and $V_{\mathrm{Te}}$. Interstitial $\mathrm{Ge}$ are strong carrier scattering centers, but they should be much less frequent than germanium incorporated on either Ga or Te sites, as seen in
Figs. 12. Most other extrinsic defects considered are moderate to low scatterers.

\section{ANALYSIS}

The results in Fig. 15 must be analyzed in conjunction with the results from the previous sections. In order to optimize the performance of the crystal as a radiation detector, we should favor:

1. defects with lower charge states (neutral) instead of ones in higher charge states.

2. charged defects that introduce shallow levels instead of deep ones.

3. defects that introduce less structural distortions (weak carrier scatterers).

In addition, we seek to compensate intrinsic free carriers in the material through dopants, respecting the precepts stated above as much as possible.

For example, despite being strong carrier scatterers, $\mathrm{Te}_{i}$ are neutral across the band gap of GaTe, so they do not add free 

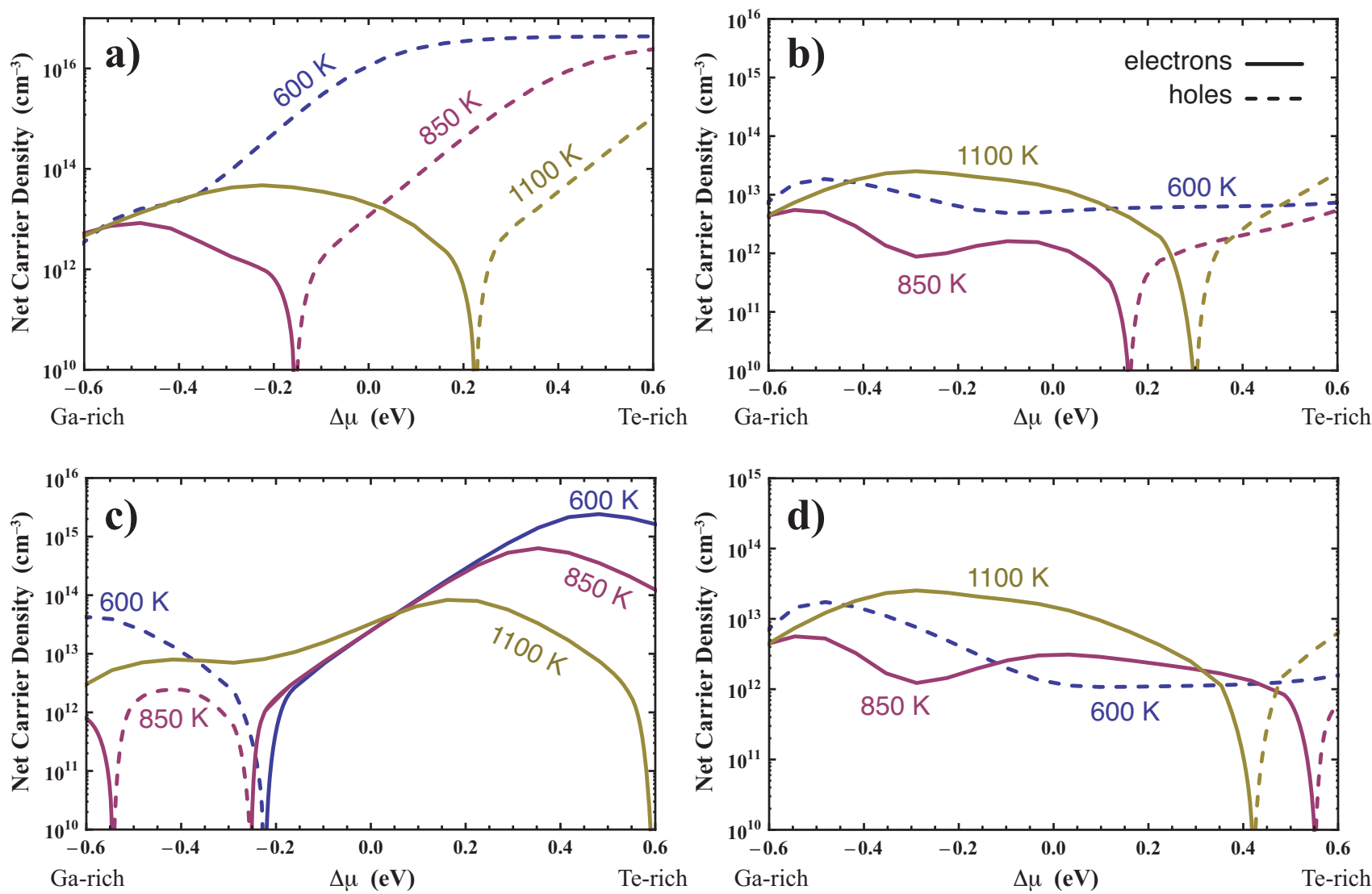

FIG. 14: (Color online) Same as Fig. 13(d), but including Ge and In impurities as well. (a) Ge: $5 \times 10^{16} \mathrm{~cm}^{-3}$, (b) $\mathrm{Ge}: 10^{17} \mathrm{~cm}{ }^{-3}$, (c) Ge: $5 \times 10^{17} \mathrm{~cm}^{-3}$, (d) Ge: $10^{16} \mathrm{~cm}^{-3}$, In: $10^{19} \mathrm{~cm}^{-3}$. The concentration of $\mathrm{H}$ and $\mathrm{O}$ impurities, respectively, is $10^{17}$ and $10^{19} \mathrm{~cm}^{-3}$ in all cases.

carriers or intra-gap levels. However, another defect that is favored under Te-rich growth situation, $\mathrm{Te}_{\mathrm{Ga}}$ antisites, introduce two deep levels in the gap of GaTe (see Fig. 9), in spite of being electronically self compensating and not introducing free carriers, as shown in Fig. 5(c). These defects are moderate carrier scattering centers. On the other hand $\mathrm{Ga}_{\mathrm{Te}}$ is an acceptor that introduces only one deep level in the crystal and is a much weaker carrier scatterer than the other two defects just mentioned. Gallium interstitials are the second weakest carrier scatterers among the intrinsic defects, after $\mathrm{Ga}_{\mathrm{Te}}$. Gallium interstitials are also the only native donors observed in GaTe [see Fig. 5(c)]. The frequency with each defect occurs is given by Eq. (5). The carrier scattering rate goes with the square of the scattering strength $M$ (Eq. (9)), indicated in Fig. 15.

These results indicate that a Ga-rich growth environment is preferred, since it reduces the presence of carrier traps associated with defects such as $\mathrm{Te}_{\mathrm{Ga}}$ and $V_{\mathrm{Ga}}$, which along with $\mathrm{Te}_{i}$ also happen to scatterer carriers much stronger than defects which are prevalent under Ga-rich conditions, such as $\mathrm{Ga}_{\mathrm{Te}}$ and $\mathrm{Ga}_{i}$. In addition, $\mathrm{Ga}_{i}$ is the only intrinsic donor we found, and its presence can result in better compensated material with higher resistivity.

Regarding extrinsic impurities, we see in Fig. 15 that $\mathrm{O}$ impurities, despite introducing moderate carrier scattering centers, are mostly electronically inert. Therefore, unless $\mathrm{O}$ is present in amounts large enough to cause formation of competing phases to $\mathrm{GaTe}^{39}$ (see Sect. IIID), its presence is not a major limiting factor for the performance of GaTe radiation detectors. Hydrogen, on the other hand, is not such a strong carrier scatterer, but it is a strong trap for electrons, as discussed in Sect. III B. Thus, $\mathrm{H}$ is much more detrimental to the crystal's electronic properties than O. Since the most favorable configuration for $\mathrm{H}$ impurities in GaTe is a very mobile interstitial, it would be extremely difficult to limit its occurrence even if the crystal is grown in a highly pure chamber. Incorporation of $\mathrm{H}$ is also likely to occur during operation of the device, particularly if specially-designed encapsulation is not employed.

Doping with In does not add significant carrier scattering to the crystal, nor does substitutional $\mathrm{Ge}$ (on either $\mathrm{Ga}$ or $\mathrm{Te}$ sites). On interstitial sites, however, Ge becomes a strong carrier scatterer, however it does not introduce significant numbers of free carriers to the crystal. However, as shown in Sect. III C, under Ga-rich conditions and for nearly intrinsic or compensated material (Fermi level close to the middle of the band gap), the formation energy of these interstitials is about $0.5 \mathrm{eV}$ higher than the substitutional configurations on Te or Ga sites. In fact, the Fermi level can be pinned near the middle of the gap by the incorporation of substitutional Ge dopants on both sites, as shown in Fig. 12 and discussed 


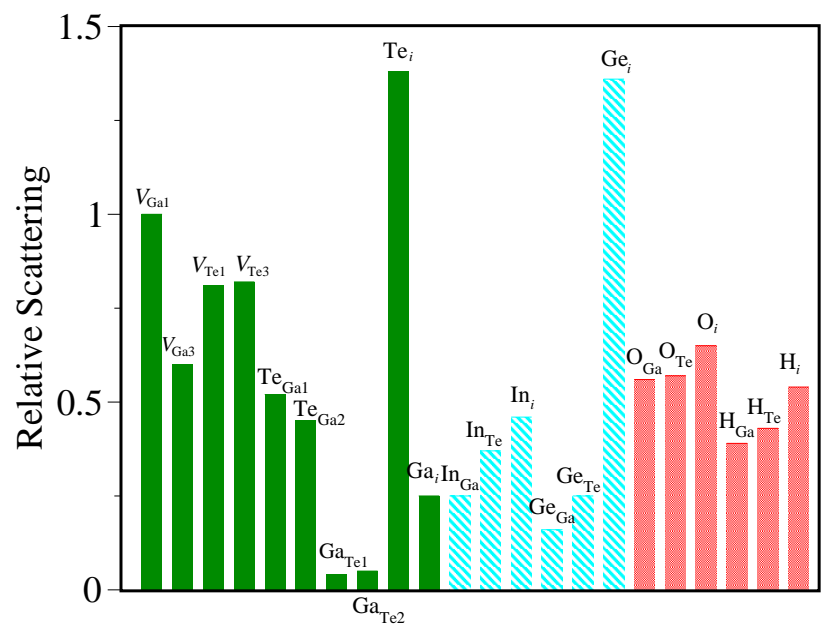

FIG. 15: (Color online) Relative average scattering strengths of intrinsic defects (green solid bars), impurities (blue dashed bars ) and dopants (red shaded bars) in GaTe. Only the lowest formation energy configurations are shown for $\mathrm{Ga}$ and Te interstitials (site $I_{1}$ ) and for extrinsic defects. The value for $V_{\mathrm{Ga} 1}$ was set as unity.

in Sect. IIIC. These results indicate that both In and Ge are good candidates for compensation doping of GaTe. In addition to their advantageous properties described above, neither one would significantly degrade charge transport in the crystal.

\section{v. CONCLUSIONS}

Given its complex and layered structure, even perfect crystalline GaTe presents highly dissimilar electronic properties in different directions, with carriers almost six times heavier along one of the in-plane directions (perpendicular to the horizontal Ga-Ga bonds shown in Fig. 3) than in the other in-plane direction, as well as across the atomic layers. Considering the highly anisotropic effective masses and the fact that the carrier mobilities across the stack of atomic layers are dominated by homopolar optical phonon scattering, one should carefully choose where to place the electrical contacts with respect to crystallographic directions when fabricating devices for detectors or for characterization measurements. Also due to the anisotropy of its crystal structure, $\mathrm{GaTe}$ is a material prone to several intrinsic defects. Moreover, its highly anisotropic structure can cause defects on different sites to present markedly different electronic behavior. As a consequence, different electronic gap states identified experimentally can be associated with a single transition of a given defect, but with the defect in different symmetry environments of the crystal. The $V_{\mathrm{Ga}}^{2-/-}$ and $\mathrm{Te}_{\mathrm{Ga}}^{+/-}$are important examples.

The most frequent native defects are Te and Ga interstitials, respectively, for crystals grown under Ga-rich or Te-rich conditions. The $\mathrm{Te}_{i}$, although electronically inactive, is the strongest carrier scatterer (most detrimental to carrier mobili- ties) found in our analysis, while the $\mathrm{Ga}_{i}$ is a relatively weak carrier scatterer and is the only intrinsic donor identified in GaTe. The $\mathrm{Te}_{\mathrm{Ga}}$ antisites that also are prevalent in Te-rich material are moderate carrier scatterers and also introduce at least two deep levels into the material (detrimental to carrier lifetimes). Gallium-rich crystals, on the other hand, have a higher concentration of $\mathrm{Ga}_{\mathrm{Te}}$ antisites, which are weak carrier scatterers and only introduce one deep and one shallow level in the band gap. Moreover, Ga-rich crystals have higher concentrations of $\mathrm{Ga}_{i}$ which are the only donors identified among the intrinsic defects analyzed. Their presence help compensate acceptor-type impurities which have invariably have been reported experimentally to-date. Finally, shifting the stoichiometric balance towards Ga-rich conditions reduces the occurrence of $V_{\mathrm{Ga}}$, which are strong carrier scatterers and also introduce deep levels. Our analysis strongly suggests that Garich material should have significantly higher resistivity, carrier mobilities, and carrier lifetimes compared to Te-rich material.

As Te is a chalcogen, contamination by $\mathrm{O}$ atoms in GaTe is expected to happen easily. This is not a major concern, since $\mathrm{O}$ is electrically inactive and also a weaker carrier scatterer than most intrinsic defects in GaTe, thus neither resistivity nor mobility is strongly affected by $\mathrm{O}$ impurities. Hydrogen impurities, on the other hand, are a major concern, as they contribute strong shallow acceptor states that are suspected to at least account partly for the $p$-type character always observed in as-grown GaTe. Since $\mathrm{H}$ tends to incorporate in interstitial sites on GaTe, it is probably insufficient to control the purity of the chamber during growth to prevent the occurrence of $\mathrm{H}$ contamination; it is likely to happen during operation of the device, upon exposure to the atmosphere.

Although In is mostly electronically inactive in GaTe, its presence can be beneficial for achieving high quality GaTe for radiation detectors. Indium can form several compounds with excess Te atoms, shifting the chemical equilibrium during crystal growth towards more Ga-rich conditions, consequently decreasing the occurrence of detrimental $V_{\mathrm{Ga}}, \mathrm{Te}_{\mathrm{Ga}}$ and $\mathrm{Te}_{i}$ defects.

Germanium doping — due to its ambipolar electronic behavior in GaTe, being an acceptor on Te sites and a donor on $\mathrm{Ga}$ sites - can be an effective way to compensate the material and increase resistivity, particularly if a carefully controlled annealing process can be used to tune the chemical environment of the dopants. Incorporation of $\mathrm{Ge}$, as well as incorporation of In, does not significantly degrade charge transport in the crystal by introducing carrier scattering.

\section{Acknowledgments}

This work performed under the auspices of the U.S. Department of Energy by Lawrence Livermore National Laboratory under Contract DE-AC52-07NA27344, with support from the National Nuclear Security Administration Office of Nonproliferation Research and Development (NA-22). 
* Electronic address: rochaleaolellnl.gov

$\dagger$ Electronic address: lordi2@llnl.gov

1 T. E. Schlesinger, J. E. Toney, H. Yoon, E. Y. Lee, B. A. Brunett, L. Franks, and R. B. James, Mat. Sci. and Eng.: R: Reports 32, 103 (2001).

${ }^{2}$ P. J. Sellin, Nuclear Instruments and Methods in Physics Research Section A: Accelerators, Spectrometers, Detectors and Associated Equipment 513, 332 (2003).

${ }^{3}$ K. Vetter, Ann. Rev. of Nuc. and Part. Sci. 57, 363 (2007).

${ }^{4}$ C. Tatsuyama, Y. Watanabe, C. Hamaguchi, and J. Nakai, J. of the Phys. Soc. of Jap. 29, 150 (1970).

5 A. Gouskov, J. Camassel, and L. Gouskov, Prog. in Crystal Growth and Characterization 5, 323 (1982).

6 Y. Cui, D. D. Caudel, P. Bhattacharya, A. Burger, K. C. Mandal, D. Johnstone, and S. A. Payne, J. of App. Phys. 105, 053709 (2009).

${ }^{7}$ H. S. Gder, B. Abay, H. Efeoglu, and Y. K. Yogurtu, Journal of Luminescence 93, 243 (2001).

8 S. Shigetomi, T. Ikari, and H. Nakashima, Jap. Jour. of App. Phys. Part 1 37, 3282 (1998).

${ }^{9}$ S. Pal and D. N. Bose, Sol. Stat. Comm. 97, 725 (1996).

10 V. Lordi, P. Erhart, and D. Åberg, Phys. Rev. B 81, 235204 (2010).

11 D. Åberg, P. Erhart, A. J. Williamson, and V. Lordi, Phys. Rev. B 77, 165206 (2008).

12 P. Erhart, D. Åberg, and V. Lordi, Phys. Rev. B 81, 195216 (2010).

13 P. Hohenberg and W. Kohn, Phys. Rev. 136, B864 (1964).

${ }^{14}$ W. Kohn and L. J. Sham, Phys. Rev. 140, A1133 (1965).

15 D. M. Ceperley and B. J. Alder, Phys. Rev. Lett. 45, 566 (1980).

16 P. E. Blöchl, Phys. Rev. B 50, 17953 (1994).

17 G. Kresse and D. Joubert, Phys. Rev. B 59, 1758 (1999).

18 G. Kresse and J. Hafner, Phys. Rev. B 47, 558 (1993).

19 G. Kresse and J. Hafner, Phys. Rev. B 49, 14251 (1994).

${ }^{20}$ G. Kresse and J. Furthmüller, Phys. Rev. B 54, 11169 (1996).

${ }^{21}$ G. Kresse and J. Furthmüller, Comput. Mater. Sci. 6, 15 (1996).

${ }^{22}$ M. Julien-Pouzol, S. Jaulmes, M. Guittard, and F. Alapini, Acta
Crystallographica Section B 35, 2848 (1979).

23 J. P. Perdew, K. Burke, and M. Ernzerhof, Phys. Rev. Lett. 77, 3865 (1996).

24 J. Sanchez-Royo, J. Pellicer-Porres, A. Segura, V. MunozSanjose, G. Tobias, P. Ordejon, E. Canadell, and Y. Huttel, Phys. Rev. B 65 (2002).

25 S. Rak, S. D. Mahanti, K. C. Mandal, and N. C. Fernelius, J. Phys.: Condens. Matter 21, 015504 (2009).

${ }^{26}$ G. Ottaviani, C. Canali, and F. Nava, Solid Stat. Commun. 14, 933 (1974).

27 L. Gouskov and A. Gouskov, Solid Stat. Commun. 28, 99 (1978).

${ }^{28}$ G. Makov and M. C. Payne, Phys. Rev. B 51, 4014 (1995).

29 S. B. Zhang and J. E. Northrup, Phys. Rev. Lett. 67, 2339 (1991).

${ }^{30}$ C. Persson, Y.-J. Zhao, S. Lany, and A. Zunger, Phys. Rev. B 72, 035211 (2005).

31 Z. H. Levine and D. C. Allan, Phys. Rev. Lett. 63, 1719 (1989).

32 X. Gonze and C. Lee, Phys. Rev. B 55, 10355 (1997).

33 A. Alkauskas, P. Broqvist, and A. Pasquarello, Phys. Rev. Lett. 101, 046405 (2008).

${ }^{34}$ P. Rinke, A. Janotti, M. Scheffler, and C. G. Van de Walle, Phys. Rev. Lett. 102, 026402 (2009).

35 M. Fiederle, C. Eiche, M. Salk, R. Schwarz, K. Benz, W. Stadler, D. Hofmann, and B. Meyer, J. Appl. Phys. 84, 6689 (1998).

${ }^{36}$ P. Erhart, D. Åberg, B. W. Sturm, K.-J. Wu, and V. Lordi, Appl. Phys. Lett. 97, 142104 (2010).

37 A. M. Conway, C. E. Reinhardt, R. J. Nikolic, A. J. Nelson, T. F. Wang, K. J. Wu, S. A. Payne, A. Mertiri, G. Pabst, R. Roy, et al., pp. 1551-1555 (2007), IEEE Nuclear Science Symposium/Medical Imaging Conference, Honolulu, HI, OCT 26-NOV 03, 2007.

38 C. Cevdet and H. Efeoglu, Semiconductor Sci. and Tech. 18, 23 (2003).

39 M. Dutreilh, P. Thomas, J. C. Champarnaud-Mesjard, and B. Frit, Solid State Sciences 3, 423 (2001). 